ARTICLE

DOI: $10.1038 / s 41467-018-03412-8$

\title{
A conserved interaction of the dynein light intermediate chain with dynein-dynactin effectors necessary for processivity
}

\author{
In-Gyun Lee ${ }^{1}$, Mara A. Olenickํ․ Malgorzata Boczkowska', Clara Franzini-Armstrong², Erika L.F. Holzbaur ${ }^{1}$ \\ $\&$ Roberto Dominguez ${ }^{1}$
}

Cytoplasmic dynein is the major minus-end-directed microtubule-based motor in cells. Dynein processivity and cargo selectivity depend on cargo-specific effectors that, while generally unrelated, share the ability to interact with dynein and dynactin to form processive dynein-dynactin-effector complexes. How this is achieved is poorly understood. Here, we identify a conserved region of the dynein Light Intermediate Chain 1 (LIC1) that mediates interactions with unrelated dynein-dynactin effectors. Quantitative binding studies map these interactions to a conserved helix within LIC1 and to N-terminal fragments of Hook1, Hook3, BICD2, and Spindly. A structure of the LIC1 helix bound to the N-terminal Hook domain reveals a conformational change that creates a hydrophobic cleft for binding of the LIC1 helix. The LIC1 helix competitively inhibits processive dynein-dynactin-effector motility in vitro, whereas structure-inspired mutations in this helix impair lysosomal positioning in cells. The results reveal a conserved mechanism of effector interaction with dynein-dynactin necessary for processive motility.

\footnotetext{
${ }^{1}$ Department of Physiology, Perelman School of Medicine, University of Pennsylvania, Philadelphia, PA 19104, USA. ${ }^{2}$ Department of Cell and Developmenta Biology, University of Pennsylvania, Philadelphia, PA 19104, USA. Correspondence and requests for materials should be addressed to

R.D. (email: droberto@pennmedicine.upenn.edu)
} 
C ytoplasmic dynein 1 (dynein) is the major minus-enddirected microtubule-based motor in eukaryotic cells. It is responsible for the transport of very diverse cargoes from the periphery to the center of the cell, including lysosomes, mitochondria and autophagosomes ${ }^{1-4}$. Recent work has shown that both cargo-specificity and processivity depend on the interaction of dynein with its general adaptor, the dynactin complex, and a series of cargo-specific effectors, including BICD2 ${ }^{5-7}$, Hook $1 / 3^{5,8,9}$, Spindly ${ }^{5}$, FIP3 ${ }^{5}$ and NIN/NINL ${ }^{10}$. These proteins are generally unrelated at the sequence level, but they all contain large portions of predicted coiled-coil structure and share the ability to interact with both dynein and dynactin to activate processive motility ${ }^{5,6,8,10,11}$. It remains unclear, however, whether each effector has evolved these functions independently or whether they share common structural-functional features and similar interactions with dynein and dynactin. Here, we show that a conserved amphipathic helix within the unstructured Cterminal region of the dynein Light Intermediate Chain 1 (LIC1) interacts with diverse dynein-dynactin effectors. The interactions were quantitatively characterized using purified proteins and isothermal titration calorimetry (ITC). A crystal structure of the LIC1 helix in complex with the N-terminal Hook domain of Hook3 reveals a conformational change within the Hook domain that gives rise to a hydrophobic cleft where the LIC1 helix binds. Supporting the importance of the LIC1-effector interaction, we found that the LIC1 helix competitively inhibits the processive motility of dynein-dynactin in complex with either Hook3 or BICD2 in single-molecule assays using total internal reflection fluorescence (TIRF) microscopy. Finally, in cellular assays, mutating the LIC1 helix leads to defective dynein-driven positioning of lysosomes. Together, the results reveal the existence of a conserved mechanism of interaction between functionally unrelated dynein-dynactin effectors and the dynein LIC1, which is required for processive dynein-driven transport.

\section{Results}

Hook interacts with the dynein LIC1 via the Hook domain. The dynein LICs, comprising two closely related isoforms (LIC1 and LIC2), consist of two domains-an N-terminal GTPase-like domain that interacts with the dynein heavy chain ${ }^{11}$ and a less conserved and predicted unstructured C-terminal region, referred to here as the effector-binding domain. Using pull-down studies, it had been previously shown that the LIC1-effector-binding domain interacts with several dynein-dynactin effectors, including Hook3, FIP3, BICD2, and Spindly ${ }^{9,11,12}$. On the other hand, a group of dynein-binding proteins, including BICD2, Spindly, HAP1, and TRAK share a coiled-coil segment, termed the CC1Box, that has been directly implicated in LIC1 binding ${ }^{12}$. Here, we set out to specifically map and quantitatively characterize the interactions of LIC1 with several dynein-dynactin effectors, including Hook1, Hook3, BICD2, and Spindly.

Hook1 and Hook3 are known dynein effectors ${ }^{5,8,9}$ that function in endosomal transport ${ }^{13-15}$. We expressed truncated constructs of human Hook1 and Hook3 in E. coli, whereas fulllength Hook1 was expressed in insect cells (Fig. 1a, b). Because Hook contains several regions of predicted coiled-coil (CC1-4) (Fig. 1a), we first analyzed whether these constructs were dimeric or monomeric using light scattering. A construct corresponding to the $\mathrm{N}$-terminal Hook domain (Hook $1_{11-166}$ ) was monomeric, whereas construct Hook $1_{11-443}$, extending to the end of CC2 was dimeric (Fig. 1c). In contrast, Hook $1_{11-238}$, comprising only the globular Hook domain and CC1 region, was in equilibrium between dimers and monomers, as indicated by an experimentally measured mass of $39.7 \mathrm{kDa}$, i.e., intermediate between those of the dimer and the monomer. Full-length human LIC1 was expressed as a fusion protein with MBP to increase its solubility, and was also found to be monomeric by light scattering (Fig. 1c).

Using ITC, MBP-LIC1 $1_{\mathrm{FL}}$ bound Hook $1_{11-443}$ with low micromolar affinity $\left(K_{\mathrm{D}}=8.1 \mu \mathrm{M}\right)$ and $\sim 1: 1$ stoichiometry, i.e., two LIC1 molecules per Hook1 dimer (Fig. 1d). Note that this ITC titration was performed at $30{ }^{\circ} \mathrm{C}$, instead of $20^{\circ} \mathrm{C}$ for most titrations performed here, because the amount of heat given off by this reaction was too small to allow for reliable fitting of the thermodynamic parameters. Consistent with the light scattering results, the titration of Hook $1_{11-238}$ into buffer produced a significant endothermic reaction, which was interpreted as indicative of dimer dissociation, with a $K_{\mathrm{D}}$ of $2.1 \mu \mathrm{M}$ (Fig. 1e). This conclusion was confirmed by analysis of Hook $1_{1-239}$ GCN4, a dimeric construct stabilized through the addition of a GCN4 leucine zipper at the $\mathrm{C}$ terminus, whose titration into buffer did not produce any significant heat change (Fig. 1e). Hook $1_{1-239}$ GCN4-bound MBP-LIC1 $1_{\mathrm{FL}}$ with a $K_{\mathrm{D}}$ of $12.9 \mu \mathrm{M}$ and $\sim 1: 1$ stoichiometry (Fig. $1 \mathrm{f}$ ), which is very similar to what was observed with Hook $1_{11-443}$ (Fig. 1d) despite the fact that the titration was inverted by placing Hook $1_{1-239}$ GCN4 in the syringe and $\mathrm{MBP}-\mathrm{LIC1}_{\mathrm{FL}}$ in the cell. The monomeric construct Hook $1_{11}$ 166 also bound MBP-LIC1 $1_{\mathrm{FL}}$ with similar affinity $\left(K_{\mathrm{D}}=12.7 \mu \mathrm{M}\right)$ and 1:1 stoichiometry. Together these results show that: (a) the LIC1-binding site is fully contained within the conserved $\mathrm{N}$ terminal Hook domain, (b) each Hook dimer interacts with two LIC1s, and (c) the CC1 region of Hook forms an unstable coiledcoil, which on its own cannot support stable Hook dimerization.

To gain further insights into the overall structure of Hook and the disposition of the Hook domain with respect to the coiled-coil segments, we used rotary shadowing electron microscopy to visualize full-length Hook1 (Fig. 1h). Hook had a kinesin-like appearance, with most particles displaying two well-separated globular domains at one end, connected through a short neck-like region to a long thin rod, which was often interrupted by a pronounced kink, followed by a shorter thin rod. These features were interpreted to correspond to the $\mathrm{N}$-terminal Hook domain, the unstable CC1 region, CC2, the central so-called Spindly motif $^{12}$, and CC3, respectively (Fig. 1i). The smaller C-terminal cargo-binding domain (CBD) was only occasionally visualized as a defined structural feature (Fig. 1i). This assignment of domains is consistent with the length of the segment extending from the end of the neck region to the central kink, whose mean length of

\footnotetext{
Fig. 1 Hook interacts with LIC1 via the N-terminal Hook domain. a Domain organization of Hook1 and constructs used in this study (CC: coiled-coil, CBD: cargo-binding domain). b SDS-PAGE (4-12\%) showing several of the proteins used in this study. c SEC-MALS analysis of Hook1 constructs and MBP-LIC1 $1_{F L}$ (color coded as indicated). The molar mass determined from light scattering (right $y$-axis) and the UV absorption at $280 \mathrm{~nm}$ (left $y$-axis) are plotted as a function of the elution volume. The theoretical masses are given in parenthesis. $\mathbf{d}-\mathbf{g}$ ITC titrations of LIC1 and Hook1 constructs as indicated. Listed with each titration are the concentrations of the protein in the syringe and in the cell, as well as the temperature of the experiment and parameters of the fit (stoichiometry $N$, dissociation constant $K_{\mathrm{D}}$ ). Errors correspond to the s.d. of the fits. Open symbols correspond to titrations into buffer (except part $\mathbf{e}$, where both titrations are into buffer). $\mathbf{h}$ Representative rotary shadowing EM image of Hook $1_{\mathrm{FL}}$. Scale bar, $100 \mathrm{~nm}$. White squares indicate individual Hook $1_{\mathrm{FL}}$ molecules highlighted in the zooms shown on the right. Scale bar, $50 \mathrm{~nm}$. i Close-up view of a representative Hook $\mathrm{FL}_{\mathrm{FL}}$ molecule shown alongside a cartoon representation of the Hook1 domains based on the rotary shadowing EM, secondary structure and sequence conservation analyses (see Supplementary Fig. 1). Scale bar, $50 \mathrm{~nm}$. $\mathbf{j}$ Length distribution of the region spanning from the end of the neck to the kink. Bin size, $5 \mathrm{~nm}, n=33$
} 
$\sim 31 \mathrm{~nm}$ approximately corresponds to the predicted dimensions of CC2 ( 27 nm) (Fig. 1j), as estimated from the structures of other coiled-coil proteins. The dimensions of the remaining smaller domains cannot be accurately measured at this resolution. The assignment of domains is also consistent with structural predictions and sequence conservation analyses, showing a series of coiled-coil segments (CC1, CC2, CC3, and CC4) interspersed with three globular regions (Hook domain, Spindly motif, and CBD), connected by short, unstructured loops of lower sequence conservation (Supplementary Fig. 1). The variability of the kink angle between CC2 and CC3 suggests that the regions $\mathrm{N}$ - and Cterminal to the Spindly motif move relatively independently of

a

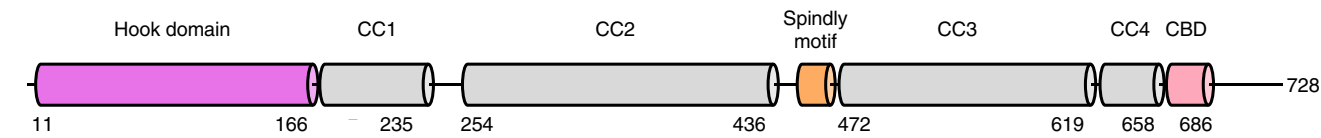

$\begin{array}{lll}\text { Hook }{ }_{11-166} & 11 \longrightarrow \\ \text { Hook } 1_{11-238} & 11 \longrightarrow & \\ & 166\end{array}$

Hook $1_{1-239 G \mathrm{CN} 4} 1$

Hook1 $_{11-443} \quad 11 \longrightarrow 443$

Hook1 $_{\mathrm{FL}}$

b

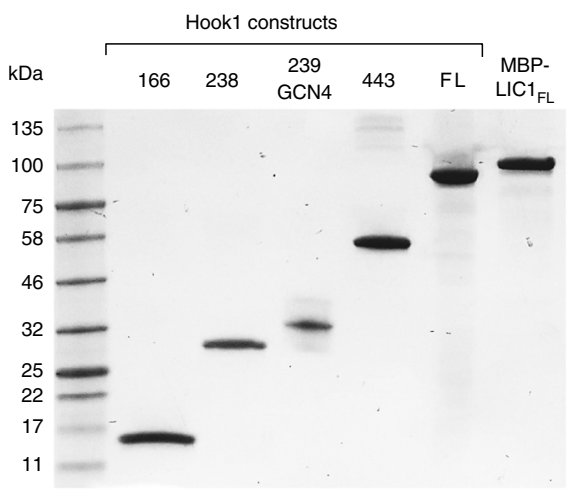

C

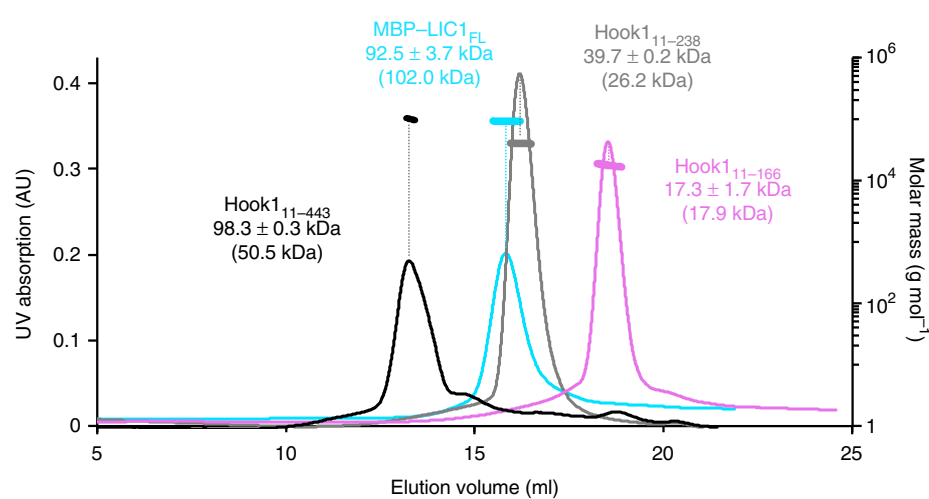

f $300 \mu \mathrm{M}$ Hook1 $1_{11-238}$
or $300 \mu \mathrm{M}$ Hook $1_{1-239}$ GCN4 $400 \mu \mathrm{M}$ MBP-LIC1 ${ }_{\mathrm{FL}}$ $\downarrow$ $20 \mu \mathrm{M}$ Hook $1_{11-443}$ Time ( $\mathrm{min})$

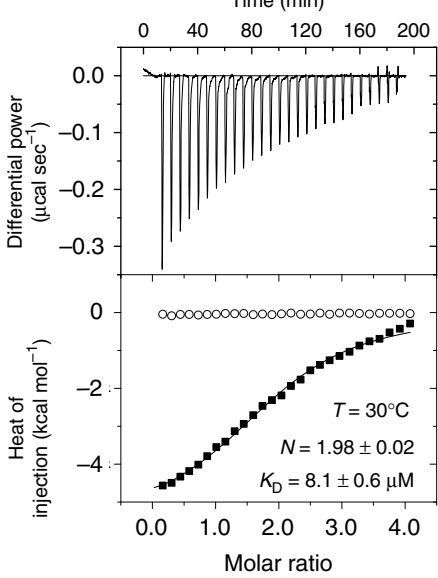

e

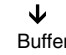

Time ( $\min )$
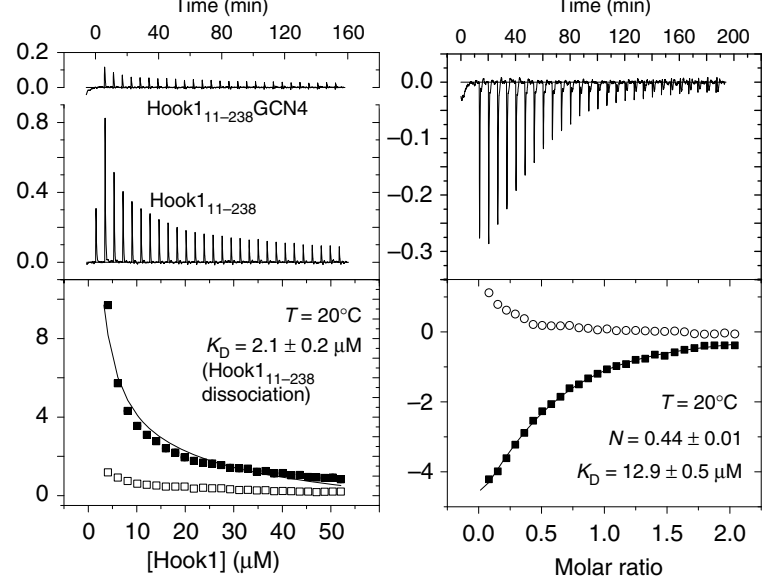

g
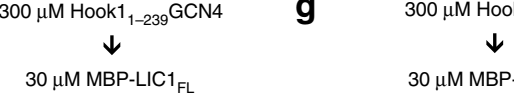

$30 \mu \mathrm{M}$ MBP-LIC1 ${ }_{\mathrm{FL}}$

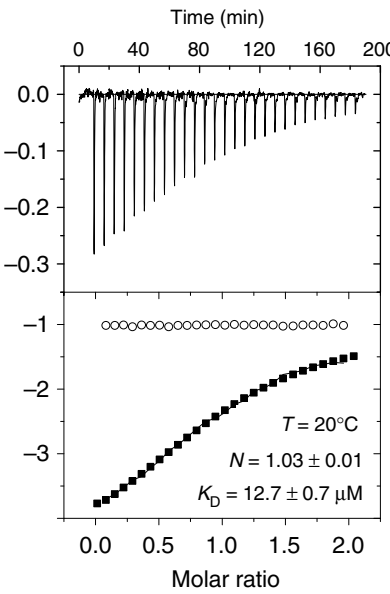

h

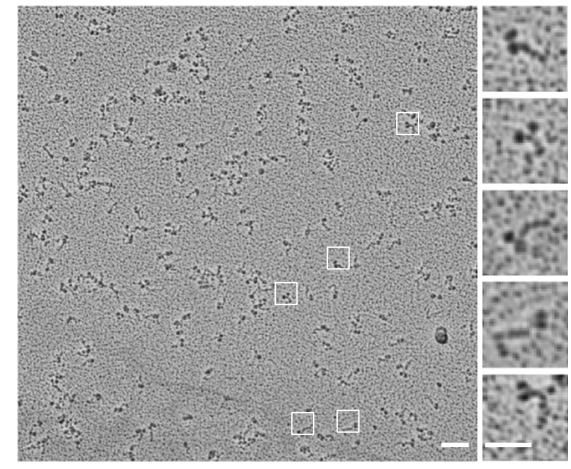

i

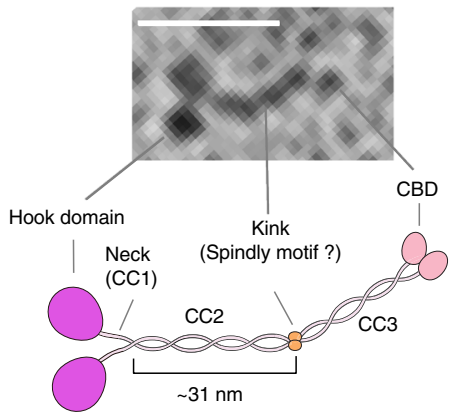

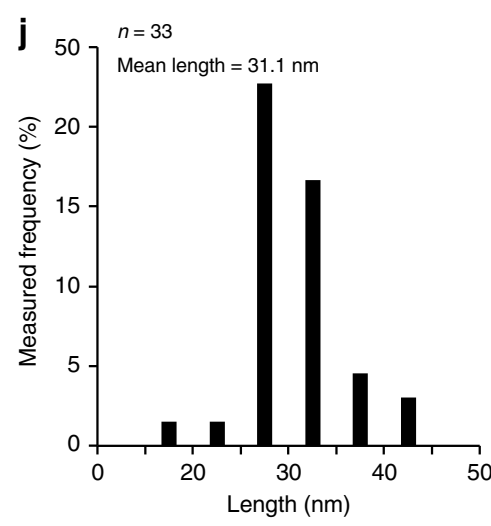


each other, i.e., the Spindly motif appears to function as a 'hinge'. Finally, the fact that the two globular Hook domains appear well separated from each other in most of the particles visualized is consistent with the two helices that form the CC1 segment (neck) not forming a stable coiled-coil, as also suggested by the light scattering (Fig. 1c) and ITC (Fig. 1e) results.
A helix in LIC1 C-terminal region binds the Hook domain. The C-terminal effector-binding domain of LIC1 (human LIC1 residues 390-523) has been shown to interact with dynein-dynactin effectors, including Hook3, BICD2, and Spindly ${ }^{9,11,12}$. However, it was unknown whether different effectors bound to the same or different regions on the LIC1 $\mathrm{C}$ terminus, and these interactions a

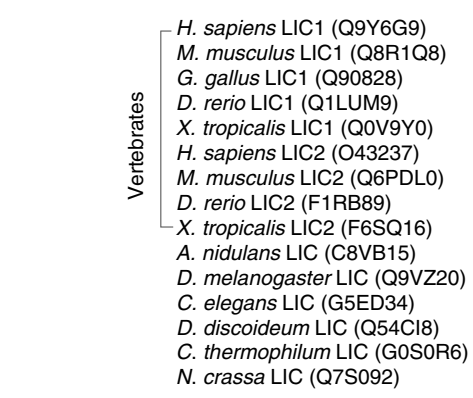

Helix-1

433437 lecele 40 lelix-1

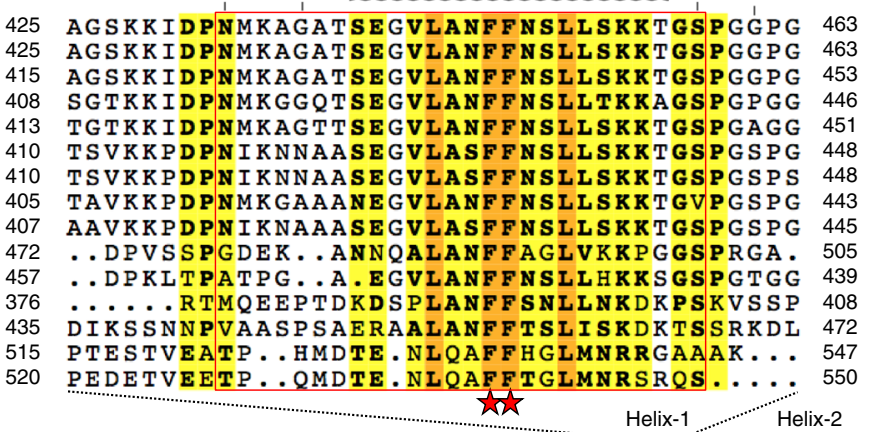

Helix-1 Helix-2

$\mathrm{LIC1} 1_{\mathrm{FL}}$

GTPase-like domain

389

Effector-binding domain

$-461$

437

$\mathrm{LIC1}_{1-437}$

LIC1 $_{\text {F447A,F448A }}$

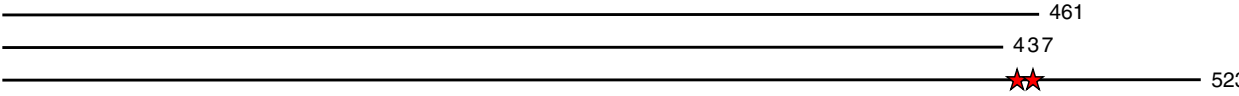

b

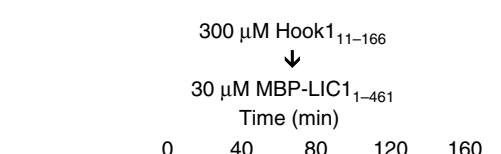

C

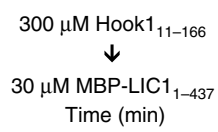

d

Time (min)
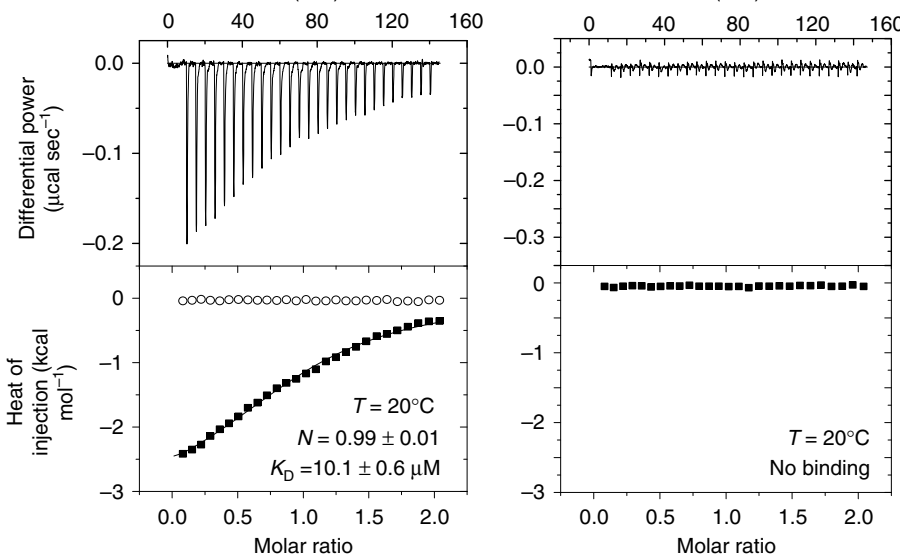

e

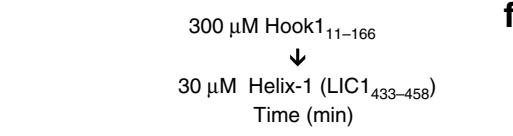

f
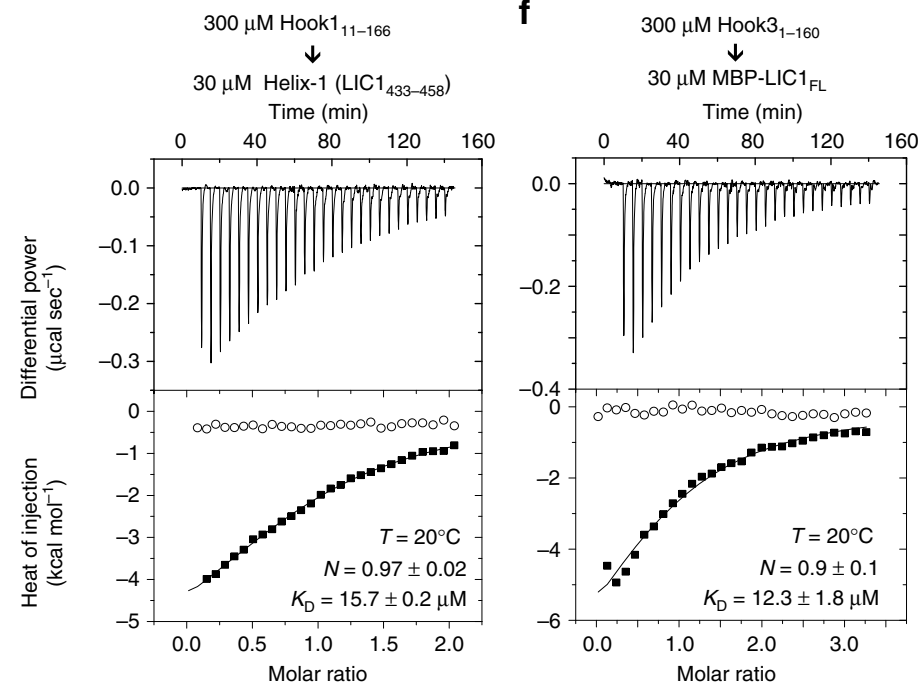

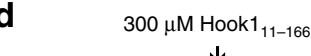

$30 \mu \mathrm{M}$ MBP-LIC1 $1_{\text {F447A,F448A }}$

Time (min)

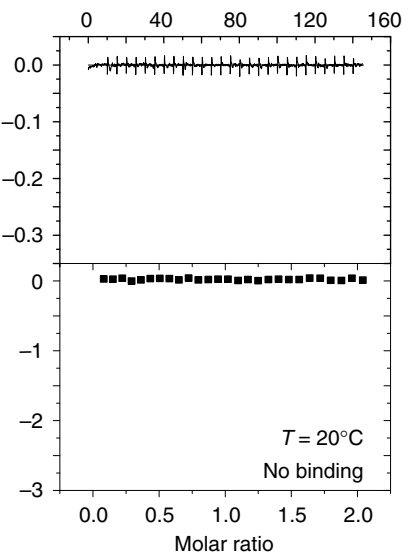

g

$300 \mu \mathrm{M} \mathrm{Hook}_{1-160}$

$30 \mu \mathrm{M}$ Helix-1 $\left(\mathrm{LIC1}_{433-458}\right)$

Time ( $\mathrm{min})$

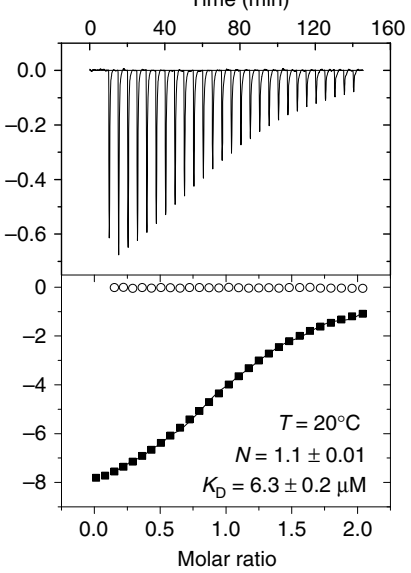


were characterized by qualitative rather than quantitative analyses. Here, we set out to map the specific region of the LIC1 C terminus implicated in interactions with Hook and other effectors (see below) and quantitatively characterize the interactions. Most of the LIC1-effector-binding domain is predicted to be unstructured and, unlike the GTPase-like domain, it is not highly conserved among species (Supplementary Fig. 2). However, sequence analysis reveals two regions of relatively high conservation that coincide with predicted $\alpha$-helical segments, which we named Helix-1 (human LIC1 residues 440-456) and Helix-2 (residues 493-502) (Fig. 2a and Supplementary Fig. 2). To test whether these conserved helical segments participate in the interaction with the Hook domain, we generated two C-terminally truncated LIC1 constructs, MBP-LIC1 $1_{1-461}$, which removes the region Cterminal to Helix-1 and MBP-LIC1 $1_{1-437}$, which additionally removes Helix-1 (Fig. 2a). MBP-LIC1 $1_{1-437}$ failed to bind the Hook domain by ITC (Fig. 2c), whereas MBP-LIC1 $1_{1-461}$ bound the Hook domain (Fig. $2 \mathrm{~b})$ with nearly the same affinity $\left(K_{\mathrm{D}}=10.1\right.$ $\mu \mathrm{M})$ as $\mathrm{MBP}-\mathrm{LICl}_{\mathrm{FL}}\left(K_{\mathrm{D}}=12.7 \mu \mathrm{M}\right)$ (Fig. $1 \mathrm{~g}$ ). These results suggested that the binding site is contained within Helix-1. Consistent with this conclusion, the Hook domain failed to bind

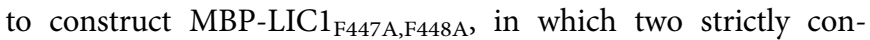
served phenylalanine residues in the middle of Helix-1 were simultaneously mutated to alanine (Fig. 2d).

To further test the role of Helix-1 in Hook binding, we expressed a 26 -a.a. peptide ( $\left.\mathrm{LIC1}_{433-458}\right)$, extending several amino acids $\mathrm{N}$ - and C-terminally to the predicted helical segment to ensure proper folding of Helix-1. The Hook domain of Hook1 bound to $\mathrm{LICl}_{433-458}$ with nearly the same affinity $\left(K_{\mathrm{D}}=15.7\right.$ $\mu \mathrm{M})$ as to MBP-LIC1 $1_{\mathrm{FL}}$ (Figs. $1 \mathrm{~g}$ and $2 \mathrm{e}$ ). The Hook domain of Hook3 (human Hook3 residues 1-160) also bound MBP-LIC1 $1_{\mathrm{FL}}$ and Helix-1 with similar affinities (Fig. 2f,g), and these affinities were comparable to those observed with the Hook domain of Hook1 (Figs. $1 \mathrm{~g}$ and 2e). Together, these results map the LIC1-Hook interaction to the conserved Helix-1 within the effector-binding domain of LIC1 and the N-terminal Hook domain of both Hook1 and Hook3. Furthermore, the conserved hydrophobic residues F447 and F448 within Helix-1 likely form part of the binding interface.

Structure of a complex of the Hook domain and the LIC1 helix. To further understand the mechanism of interaction between Hook and LIC1, we determined the crystal structure of human Hook $3_{1-160}$ in complex with human LIC1 Helix-1 at $1.5 \AA$ resolution (Fig. $3 \mathrm{a}-\mathrm{c}$ and Table 1 ). The electron density is well defined for Hook3 residues 10-160 and LIC1 residues 441-454 (Fig. 3b). The first nine amino acids of Hook3 and residues 433-440 and 455-458 of Helix-1 were disordered and are, thus, unlikely to participate in the interaction. As previously reported ${ }^{9}$, the Hook domain displays a canonical 7-helix calponin homology $(\mathrm{CH})$ like fold, featuring an additional helix at the $\mathrm{C}$ terminus termed helix a8. Generally, the structure superimposes well with that of the unbound Hook domain determined previously ${ }^{9}$, with an r.m. s. deviation of $1.4 \AA$ for 136 equivalent $\mathrm{Ca}$ atoms (Fig. 3d). However, the Hook domain-specific helix $\alpha 8$, which in the unbound structure is fully extended and interacts in anti-parallel fashion with the same helix from a symmetry-related molecule in the crystal, is broken into two helices ( $\alpha 8 \mathrm{a}$ and $\alpha 8 \mathrm{~b}$ ) in the current structure (Fig. 3d), giving rise to a $\mathrm{V}$-shaped hydrophobic cleft that constitutes the binding site for LIC1 Helix-1 (Fig. 3c). As predicted, the visualized portion of Helix-1 is folded as an amphipathic $\alpha$-helix, with its hydrophobic surface facing the hydrophobic cleft of the Hook domain (Fig. 3c). All the highly conserved, hydrophobic amino acids of the LIC1-effector-binding domain are directly inserted into the hydrophobic cleft of the Hook domain, including L444, F447, F448, and L451, explaining why the mutant MBP-LIC1 $1_{\mathrm{F} 447 \mathrm{~A}, \mathrm{~F} 448 \mathrm{~A}}$ failed to bind the Hook domain (Fig. 2d). The binding interface also coincides with the most highly conserved surface of the Hook domain (Fig. 3e).

The Hook domain also interacts with a second LIC1 Helix-1 from a neighboring complex in the crystal lattice (Fig. 3e). This interaction presents the less conserved, hydrophilic surface of Helix-1 to a less conserved surface on the Hook domain, which a priori is inconsistent with a native interaction. Yet, to rule out this interaction, we generated two Hook domain mutants: A138D, testing the presumed crystal packing contact, and M140D, testing the anticipated native binding site (Fig. 3f). The Hook $3_{1-160} \mathrm{~A} 138 \mathrm{D}$ mutant bound $\mathrm{MBP}-\mathrm{LIC1} 1_{\mathrm{FL}}$ with the same affinity as wild type Hook $3_{1-160}$ (compare Figs. $2 \mathrm{f}$ and $3 g$ ), whereas the Hook $3_{1-160}$ M140D mutant failed to bind MBP$\mathrm{LICl}_{\mathrm{FL}}$ (Fig. $3 \mathrm{~h}$ ), confirming that the native binding site of Helix1 is located at the interface between $\alpha 8 \mathrm{a}$ and $\alpha 8 \mathrm{~b}$, and conferring functional significance to the conformational change that splits helix $\alpha 8$ into two helices. Indeed, even in the presence of Helix-1, we obtained a second crystal form showing the reported extended conformation of helix $a 8^{9}$, but the LIC1 peptide was not bound in these crystals. To further test the importance of the conformational change in helix a8 for LIC1 binding, we generated a truncated construct, Hook $3_{1-143}$, lacking the $a 8 \mathrm{~b}$ portion of helix $\alpha 8$, i.e., the region that bends back to form the $\mathrm{V}$-shaped cleft (Fig. 3d). Hook $3_{1-143}$ failed to bind $M B P-L I C 1_{F L}$ (Fig. 3i). Collectively, these results confirm that the extended helix $a 8$ of the Hook domain, which distinguishes this domain from the canonical $\mathrm{CH}$ fold, undergoes a conformational change to produce a conserved, hydrophobic cleft for binding of the conserved LIC1 Helix-1.

The LIC1 helix binds diverse dynein-dynactin effectors. Next, we asked whether LIC1 Helix-1 was also implicated in interactions with other dynein-dynactin effectors that are generally structurally and functionally unrelated to each other. As mentioned above, a recent study found that a group of dynein-dynactin effectors share a region termed the CC1-Box that was implicated in LIC1 binding through pull-down and mutagenesis studies $^{12}$.

To test whether LIC1 Helix-1 also mediates the interaction with CC1-Box-containing effectors, we expressed N-terminal fragments of two effectors: BICD2 ${ }_{1-98}$ and Spindly ${ }_{1-142}$ (Fig. $4 \mathrm{a}$, b). These constructs extend $\mathrm{N}$ - and C-terminally from the CC1Box to include the first predicted coiled-coil segment of each

Fig. 2 The conserved Helix-1 within the LIC1-effector-binding domain binds the Hook domain. a Alignment of LIC sequences from different species and isoforms around the predicted Helix-1 within the C-terminal effector-binding domain (top) and domain diagram of human LIC1 showing the constructs used in this study (bottom). The name of each sequence includes the organism of origin and UniProt accession code. Yellow and orange backgrounds indicate $70 \%$ and $100 \%$ sequence conservation, respectively. Red stars highlight residues F447 and F448 that were mutated to alanine. The predicted Helix-1 and Helix-2, coinciding with regions of higher sequence conservation (see Supplementary Fig. 2), are highlighted in the domain diagram, and Helix-1 is also depicted above the sequence alignment. The region corresponding to the Helix-1 ( $\mathrm{LICl}_{433-458}$ ) peptide is contoured red. $\mathbf{b}-\mathbf{g}$ ITC titrations of Hook $1_{11-166}$ and Hook3 $3_{1-160}$ into LIC1 constructs (as indicated). Listed with each titration are the concentrations of the protein in the syringe and in the cell, as well as the temperature of the experiment and parameters of the fit (stoichiometry $N$, dissociation constant $K_{D}$ ). Errors correspond to the s.d. of the fits. Open symbols correspond to control titrations into buffer 
a

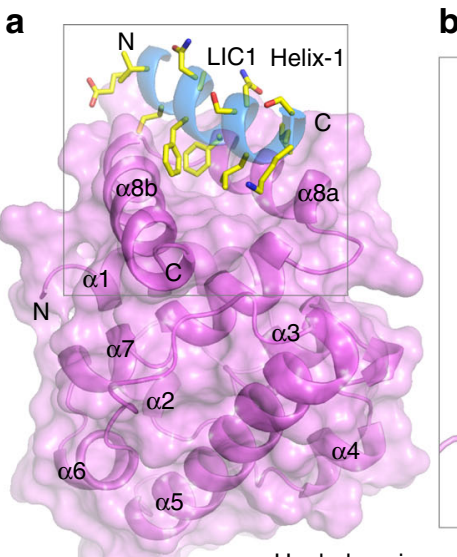

d
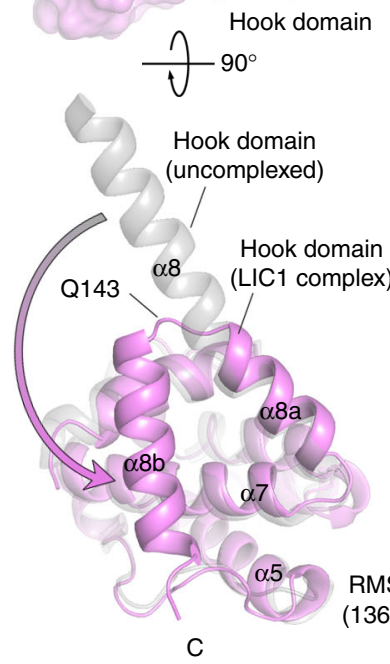

g

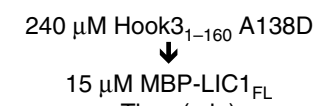

$15 \mu \mathrm{M}$ MBP-LIC1 $1_{\mathrm{FL}}$ $\begin{array}{llllll}0 & 40 & 80 & 120 & 160 & 200\end{array}$

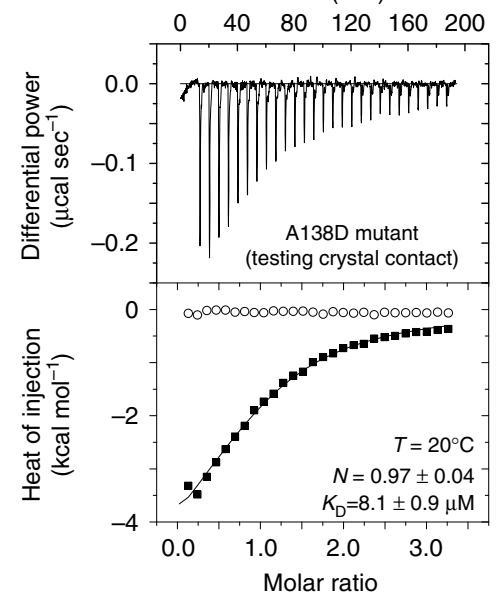

b

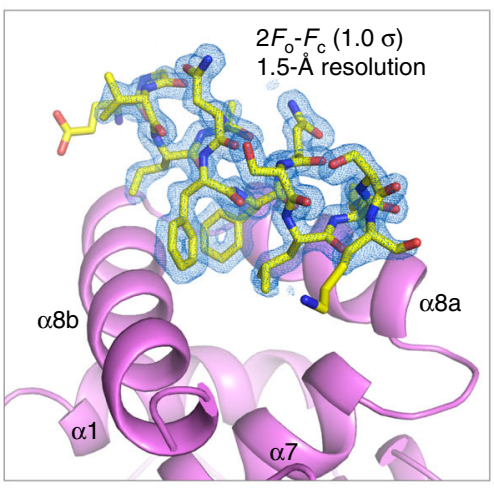

c

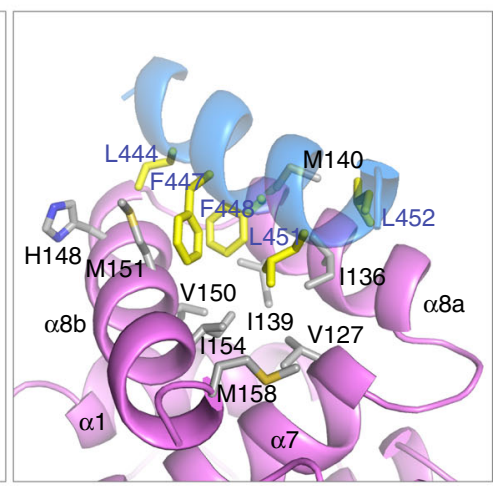

e
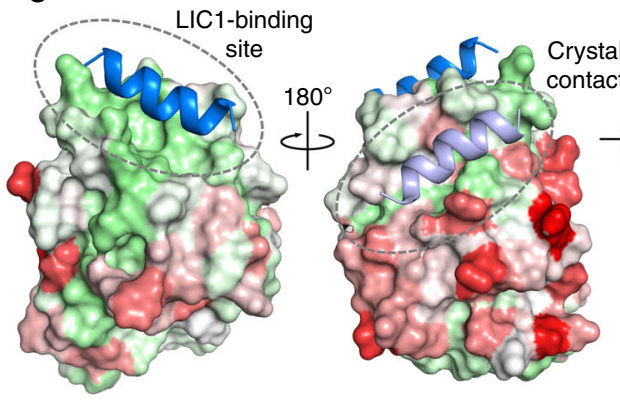

f

M140D mutant (testing LIC1-binding site)

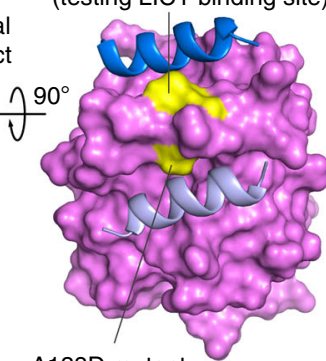

A138D mutant

(testing crystal contact)

Conservation

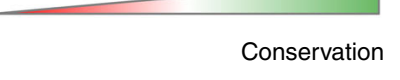

$\mathrm{RMSD}=1.4 \AA$

$136 \mathrm{C} \alpha$ atoms) h
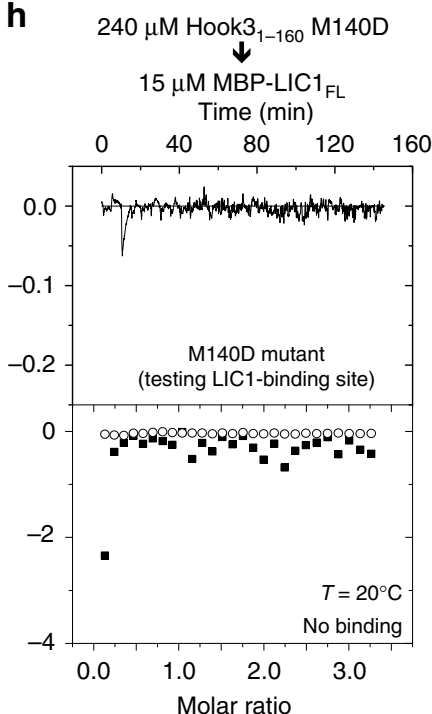

i

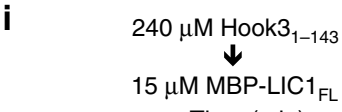

Time (min)

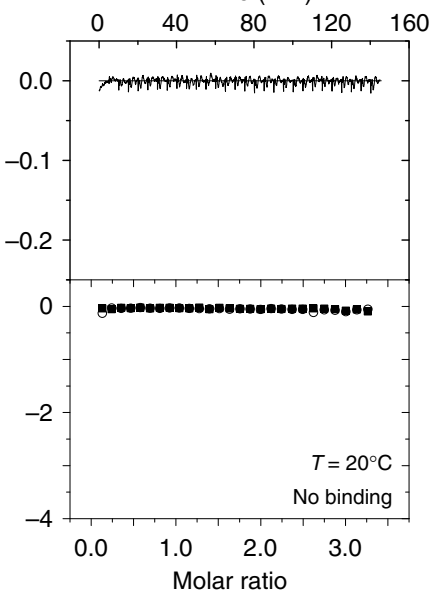

Fig. 3 Crystal structure of the Hook domain in complex with LIC1 Helix-1. a Ribbon and surface representation of the structure of Hook3 ${ }_{1-160}$ (magenta) in complex with Helix-1 ( $\mathrm{LIC1}_{433-458}$, blue). The side chains of Helix-1 are shown using a sticks representation, colored by atom type. $\mathbf{b}$ Close-up view of the Helix-1 binding site, showing the $2 F o-F c$ electron density map (blue mesh) at $1.5 \AA$ resolution, contoured at $1 \sigma$ around an all-atom representation of Helix- 1 . c Close-up view of the Helix-1 binding site, showing the residues at the hydrophobic contact interface. d Superimposition of the structure of the Hook domain from the Helix-1-bound complex (magenta) and unbound structure (gray) ${ }^{9}$. A conformational change in the C-terminal helix $\alpha 8$, which distinguishes this domain from the $\mathrm{CH}$ domain, leads to the formation of two helices ( $\alpha 8 \mathrm{a}$ and $\alpha 8 \mathrm{~b}$ ) that constitute the binding site for Helix-1. e Sequence conservation of the Hook domain (see also Supplementary Fig. 1) mapped onto the surface of the structure and colored from low to high conservation using a red to green gradient. In the crystal lattice, the Hook domain contacts a second Helix-1 from a neighboring complex (light blue). $\mathbf{f}$ Surface representation of the Hook domain (magenta), showing in yellow the two amino acids mutated (A138D and M140D) to test the functional relevance of the two Helix-1 interactions. g-i ITC titrations of the indicated Hook3 $1_{-160}$ mutants into MBP-LIC1 $1_{\mathrm{FL}}$. Experimental conditions and fitting parameters are listed. Errors correspond to the s.d. of the fits. Open symbols correspond to titrations into buffer 


\begin{tabular}{|c|c|}
\hline Data collection & \\
\hline $\begin{array}{l}\text { Space group } \\
\text { Cell dimensions }\end{array}$ & $P 2_{1} 2_{1} 2_{1}$ \\
\hline $\begin{array}{l}a, b, c(\AA) \\
\quad \alpha, \beta, \gamma\left({ }^{\circ}\right) \\
\text { Resolution }(\AA) \\
R_{\text {merge }} \\
l / \sigma l \\
\text { Completeness }(\%) \\
\text { Redundancy } \\
\text { Wilson } B \text {-factor }\left(\AA^{2}\right) \\
\text { Refinement }\end{array}$ & $\begin{array}{l}\text { 31.71, 35.37, } 126.14 \\
90.0,90.0,90.0 \\
50.00-1.50(1.55-1.50)^{\mathrm{a}} \\
0.053(0.373) \\
50.7(3.8) \\
98.7(90.9) \\
11.2(5.6) \\
14.1\end{array}$ \\
\hline $\begin{array}{l}\text { Resolution range }(\AA) \\
\text { No. of reflections } \\
R_{\text {work }} / R_{\text {free }}(\%) \\
\text { No. of non-hydrogen atoms }\end{array}$ & $\begin{array}{l}20.54-1.50(1.55-1.50) \\
23,302 \\
15.5(18.8) / 17.7(23.7)\end{array}$ \\
\hline $\begin{array}{c}\text { Protein } \\
\text { Water } \\
\text { B-factors }\left(\AA^{2}\right)\end{array}$ & $\begin{array}{l}1346 \\
121\end{array}$ \\
\hline $\begin{array}{l}\text { Protein } \\
\text { Water } \\
\text { r.m.s. deviations }\end{array}$ & $\begin{array}{l}19.4 \\
30.9\end{array}$ \\
\hline $\begin{array}{c}\text { Bond lengths }(\AA) \\
\text { Bond angles }\left({ }^{\circ}\right) \\
\text { Ramachandran }(\%)\end{array}$ & $\begin{array}{l}0.015 \\
1.490\end{array}$ \\
\hline $\begin{array}{c}\text { Favored } \\
\text { Outliers } \\
\text { PDB code }\end{array}$ & $\begin{array}{l}97 \\
0 \\
6 \mathrm{~B} 9 \mathrm{H}\end{array}$ \\
\hline
\end{tabular}

The dataset was collected from a single crystal at CHESS beamline F1 a Values in parentheses correspond to the highest resolution shell

protein and ensure proper dimerization (note that when bound to dynein-dynactin, the entire $\mathrm{N}$-terminal region of these two proteins appear to form uninterrupted coiled-coil structures ${ }^{16}$, as depicted in Fig. 4a.) Indeed, as verified by light scattering (Fig. 4c), both BICD2 $2_{1-98}$ and Spindly ${ }_{1-142}$ form stable coiled-coil dimers, with experimentally determined masses approximately double those calculated from sequence. By ITC, the titrations of $\mathrm{LIC1}_{433-458}$ into BICD2 ${ }_{1-98}$ (Fig. 4d) and Spindly ${ }_{1-142}$ (Fig. 4e) fitted best to two binding-site isotherms. The affinities of the two binding sites were similar to each other, and they were also similar for the two effectors (with $K_{\mathrm{D}} \mathrm{s}$ ranging from 1.5 to 7.6 $\mu \mathrm{M})$. Curiously, however, despite sharing a similar CC1-Box and displaying similar affinities for LIC1 Helix-1, the titrations into BICD2 ${ }_{1-98}$ and Spindly $y_{1-142}$ had different overall appearances (Fig. 4 d, e). For the Spindly ${ }_{1-142}$ titration, in particular, the two binding sites have very close affinities and are probably saturated at the same time, but the first site has a mild endothermic character, whereas the second site has a strong exothermic character, which masks the endothermic signal of the first part of the titration, explaining the peculiar shape of this reaction. Likely, LIC1 binding produces different types of conformational changes in these two proteins, which other than the CC1-Box share no apparent sequence similarity. These results confirm that LIC1 Helix-1 constitutes a common binding site for unrelated dynein-dynactin effectors, including CC1-Box-containing effectors (BICD, Spindly) and Hook-family effectors.

The LIC1 helix/effector interaction is crucial for motility. To test the functional significance of the LIC1 Helix-1 interaction with dynein-dynactin effectors, we utilized an in vitro single- molecule approach to track the movement of dynein-dynactineffector complexes obtained from cell extracts using TIRF microscopy ${ }^{8}$. Lysates of HeLa cells expressing Halo-tagged Hook3 $3_{1-552}$ labeled with TMR-HaloTag ligand were flowed into a chamber containing Taxol-stabilized microtubules immobilized on coverslips. The dynein-driven motility of single molecules was then monitored both in the absence or the presence of increasing

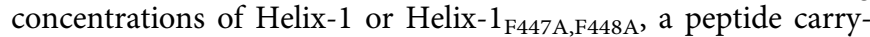
ing the two mutations found to inhibit binding of full-length LIC1 to the Hook domain (Fig. 2d). Consistent with previous reports ${ }^{5,8}$, in the absence of Helix-1 we observed robust motility of Halo-Hook3 ${ }_{1-552}$-positive complexes along microtubules, characterized by long run lengths and high velocities (Fig. 5a). In contrast, we observed a marked inhibition of processive motility with the addition of Helix-1, with nearly complete inhibition at Helix-1 concentrations of $100 \mu \mathrm{M}$ or higher, whereas the addition of Helix-1 $1_{\text {F447A,F448A }}$ did not inhibit motility (Fig. 5a). Similar results were observed in experiments that tracked the movement of dynein-dynactin-BICD2 complexes obtained from cell extracts expressing Halo-BICD2 $2_{1-572}$ labeled with TMR-HaloTag ligand (Fig. 5b). In this case, however, higher concentrations of Helix-1 $(>200 \mu \mathrm{M})$ were required for full inhibition, which is not entirely unexpected for in trans competition of an intramolecular interaction.

To assess whether the LIC1-effector interaction contributes to organelle motility in cells, we analyzed the distribution of

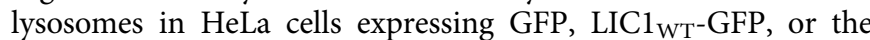
mutant $\mathrm{LIC1}_{\mathrm{F} 447 \mathrm{~A}, \mathrm{~F} 448 \mathrm{~A}}$-GFP that does not interact with Hook1 (Fig. 2d). Importantly, this mutation is predicted to also block the interaction of LIC1 with other effectors, since we found that Helix-1 is involved in interactions with several effectors (Figs. 2e, $\mathrm{g}$ and $4 \mathrm{~d}, \mathrm{e})$. Lysosomes are well-characterized cargoes of dynein, which drives perinuclear clustering of lysosomes near microtubule minus ends ${ }^{17,18}$, and LIC1 is known to be required for this activity $^{19}$. Compared to the expression of GFP alone, the expression of $\mathrm{LIC1}_{\mathrm{WT}}$-GFP did not significantly change the distribution of lysosomes, visualized by anti-LAMP1 staining of cells fixed 18-22 $\mathrm{h}$ after transfection. In contrast, the expression of the $\mathrm{LIC1}_{\mathrm{F} 447 \mathrm{~A}, \mathrm{~F} 448 \mathrm{~A}-\mathrm{GFP}}$ mutant resulted in an abnormal localization of lysosomes (Fig. 5c). In these cells, lysosomes appeared dispersed throughout the cytoplasm and did not show the characteristic perinuclear clustering seen in control cells (Fig. 5c). In a blind analysis, $\mathrm{LIC1}_{\mathrm{F} 447 \mathrm{~A}, \mathrm{~F} 448 \mathrm{~A}}$-GFP-expressing cells displayed a significantly higher percentage of abnormally positioned lysosomes compared to cells expressing GFP or LIC1 ${ }_{\mathrm{WT}}$-GFP (Fig. 5d). Together, these results show that the LIC1-effector interaction mediated by Helix-1, and specifically residues F447 and F448, is absolutely required for processive dynein-based motility in vitro and in cells.

\section{Discussion}

Cytoplasmic dynein is responsible for most cellular activities requiring microtubule minus-end-directed motility. However, in isolation, dynein is not processive $e^{5,20}$. It is now recognized that dynein's functional diversity, including cargo-specificity and processivity, depends on its interaction with the general adaptor dynactin, regulated by an ever-expanding family of dynein-dynactin effector proteins, including BICD $2^{5-9}$, Hook $1 / 3^{5,8,9}$, Spindly ${ }^{5}$, FIP $^{5}$, and NIN/NINL ${ }^{10}$. These proteins have been distinctly called adaptors ${ }^{5,9}$ or regulators ${ }^{21}$. We have used here the more general term 'effectors' because they do both-they help bring together dynein and dynactin and recruit specific cargoes, which are typical adaptor functions, but they also activate dynein processivity, thus playing a regulatory role. We have a limited understanding of how dynein-dynactin effectors exert these 
a

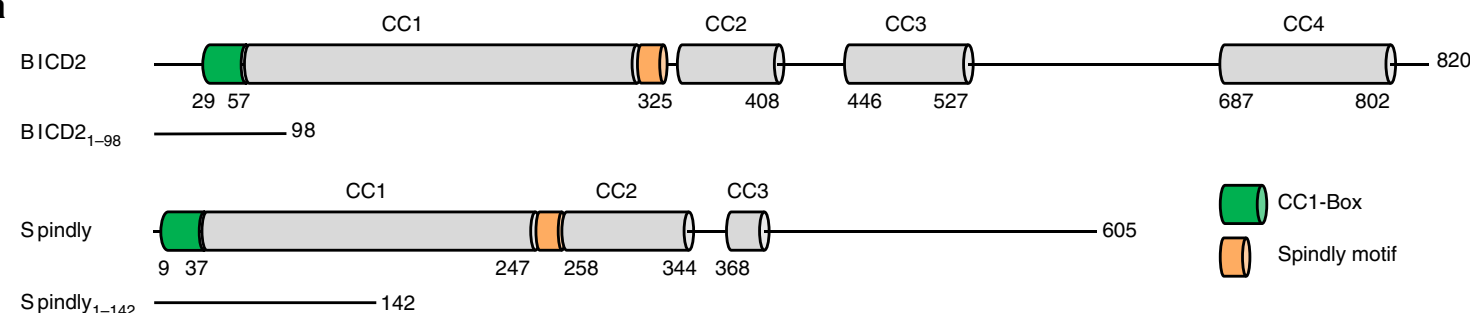

b \begin{tabular}{lr|ll} 
BICD2 & 29 & LSHELAETTREKIQAAEYGLAVLEEKHQL & 57 \\
BICD1 & 20 & LTKELTETTHEKIQAAEYGLVVLEEKLTL & 48 \\
BICDR1 & 100 & LSV. IRQKE KLVLAARLGKALLERNQMS & 127 \\
BICDR2 & 50 & LALQLQQKEKDLLLAAELGKMLLERNEEL & 78 \\
Spindly & 9 & LRCRLKEAEEERLKAAQYGLQLVESQNEL & 37 \\
HAP1 & 193 & TYGMVLQRERDLNTAARIGQSLVKNSV & 221 \\
TRAK1 & 115 & VTRLLEEKERDLELAARIGQSLLKKNKL & 143 \\
TRAK2 & 115 & VTHLLAERDRDLELAARIGQALLKRNHVL & 143
\end{tabular}

\begin{tabular}{ccc} 
Family & Function & Cargo \\
\hline BICD & $\begin{array}{c}\text { Golgi organization } \\
\text { Dynein targeting to } \\
\text { kinetochore }\end{array}$ & Rab6-vesicle \\
Spindly & RZZ complex \\
HAP1 & $\begin{array}{c}\text { Neuronal trafficking } \\
\text { TRAK }\end{array}$ & Autophagosome \\
\hline
\end{tabular}

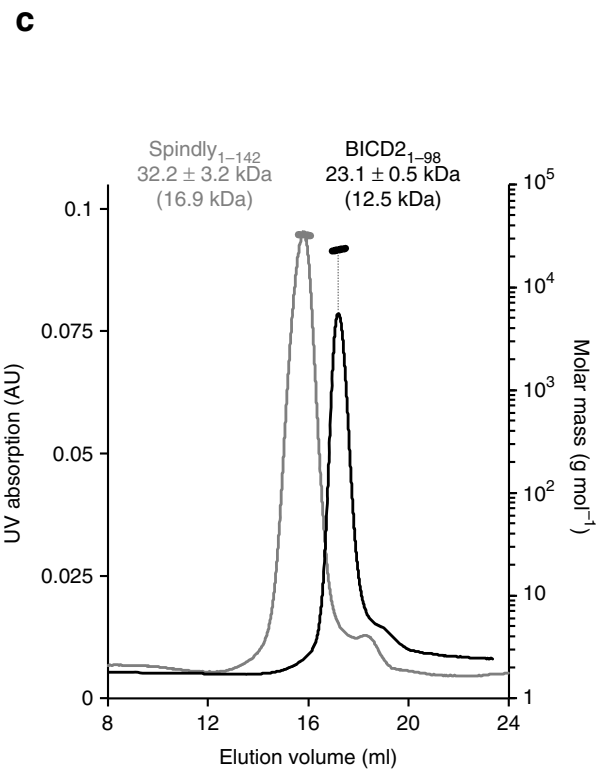

d $\quad 400 \mu \mathrm{M}$ Helix-1 $\left(\mathrm{LIC1}_{433-458}\right)$

e $500 \mu \mathrm{M}$ Helix-1 ( $\left.\mathrm{LIC1}_{433-458}\right)$
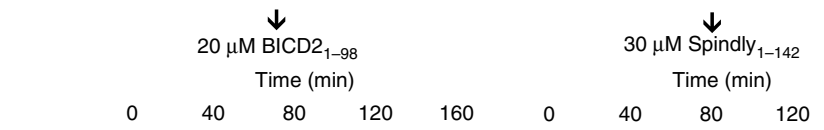

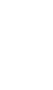


a

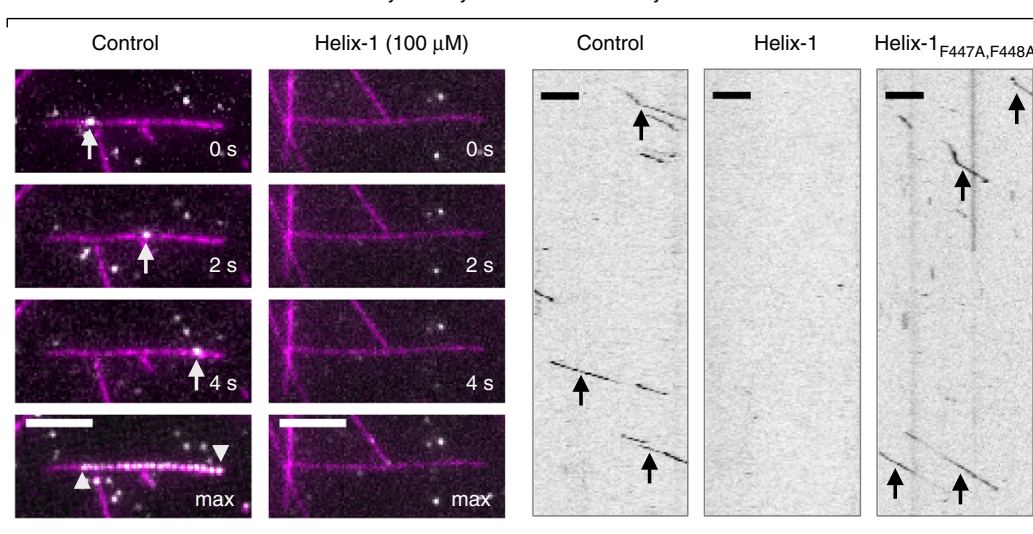

b
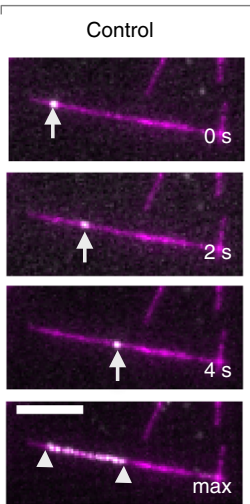

$\max$
Dynein-Dynactin-BICD2 motility

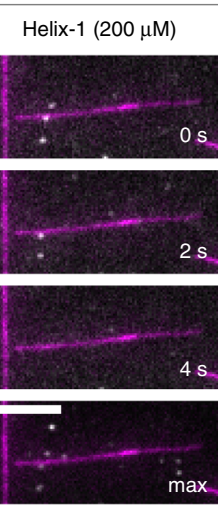

Control

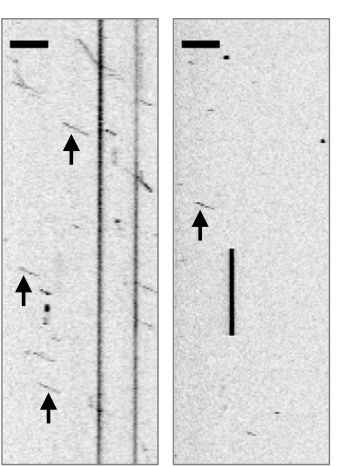

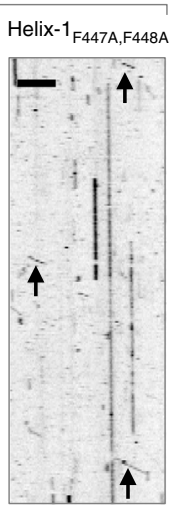
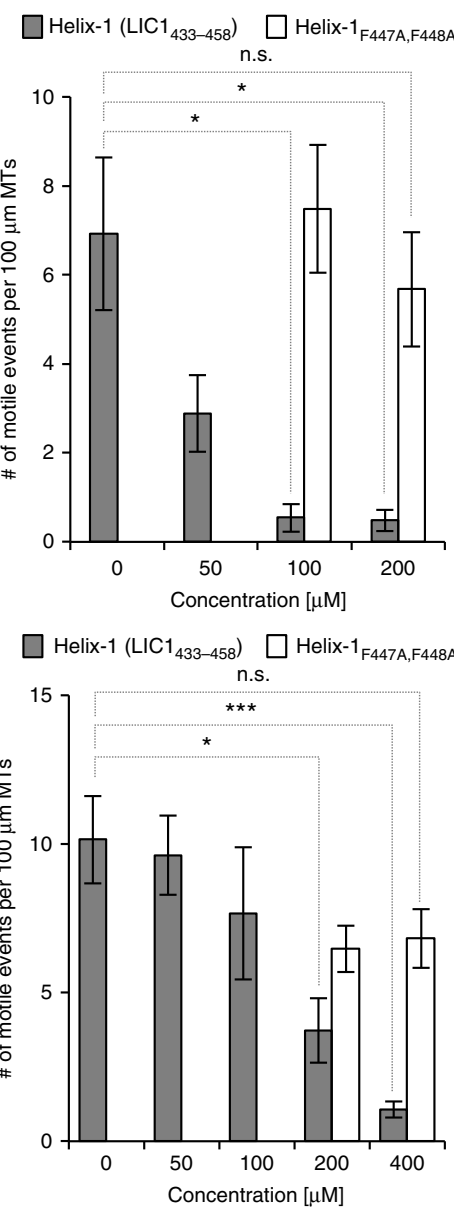

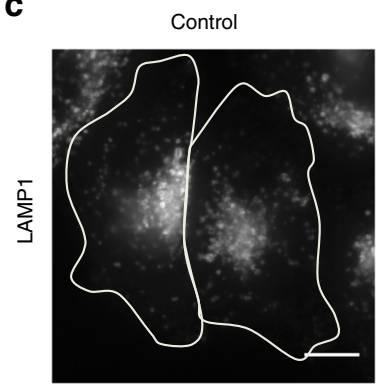

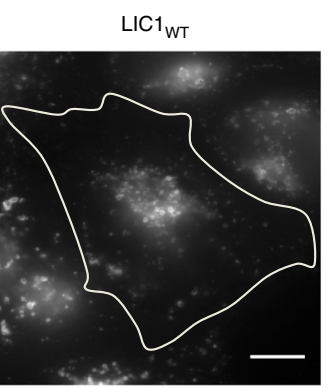

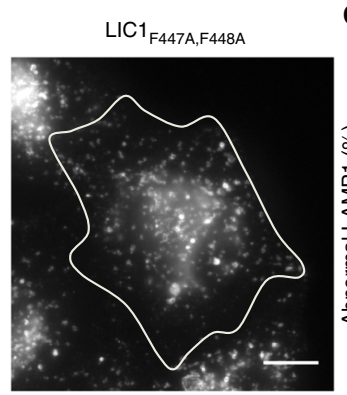

d

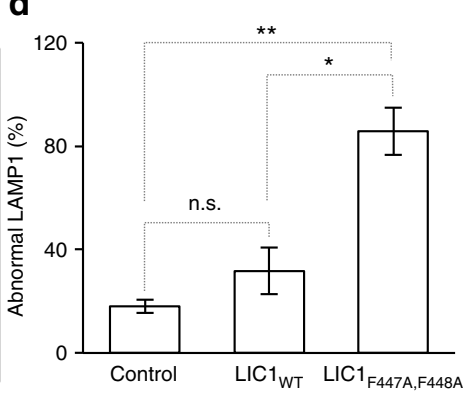

Fig. 5 The Helix-1-effector interaction is important for processive motility in vitro and in cells. a, b Time series and kymographs (1 min) of Halo-Hook31-552 and Halo-BICD2 $1-572$ runs on microtubules (magenta) in the absence (control) or the presence of Helix-1 or Helix-1 $1_{F 447 A, F 448 A}$ peptides (as indicated) analyzed by TIRF microscopy. Arrows indicate a motile particle and arrowheads indicate the beginning and end of the trajectory in a maximum projection (max). Scale bar, $5 \mu \mathrm{m}$. Quantifications (right) show that the number of motile events declines with increasing Helix-1 concentrations, but not Helix-1 ${ }_{\mathrm{F} 447 \mathrm{~A} \text {, }}$ F448A. The statistical significance of the measurements was determined using a One-way Anova test, analyzing $N=6-21$ videos and a minimum of 3 individual cell lysates per condition (n.s., non-significant; ${ }^{\star} p \leq 0.05$; ${ }^{\star \star} p \leq 0.01$; ${ }^{\star \star \star} p<0.001$ ). Error bars correspond to the s.e.m. $\mathbf{c}$ Representative images of

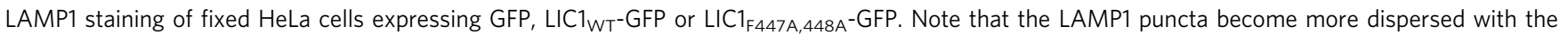
expression of $\mathrm{LIC1}_{\mathrm{F} 447 \mathrm{~A}, 448 \mathrm{~A}}-\mathrm{GFP}$, but not LIC1 $\mathrm{WT}_{\mathrm{T}}-\mathrm{GFP}$. Cell perimeters are outlined in white. Scale bar, $10 \mu \mathrm{m}$. $\mathbf{d}$ Percentage of cells with abnormal

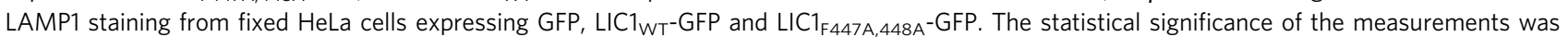

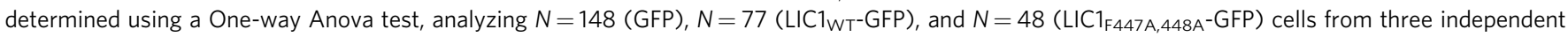
repeats (n.s., non-significant; ${ }^{*} p \leq 0.05 ;{ }^{\star \star} p \leq 0.01$ ). Error bars correspond to the s.e.m

micromolar affinities. We were also able to visualize the structural basis of this interaction at high-resolution for the Hook subfamily of effectors (comprising three isoforms, Hook1-3). Finally, we demonstrated that this interaction enhances the processive motility of dynein in vitro and that disruption of the LIC1effector interface affects organelle transport in cells. Somewhat analogous to our findings, an interaction between the light and intermediate chains of yeast dynein has been implicated in dimerization and processive motility ${ }^{34}$.

Curiously, the LIC1-effector interaction involves a conserved motif on the LIC1 side of the interface, but different surfaces on the effector side. For Hook-family effectors, the interaction involves the N-terminal Hook domain, which has a globular fold related to the $\mathrm{CH}$ domain. However, it is the extended helix a8 of 

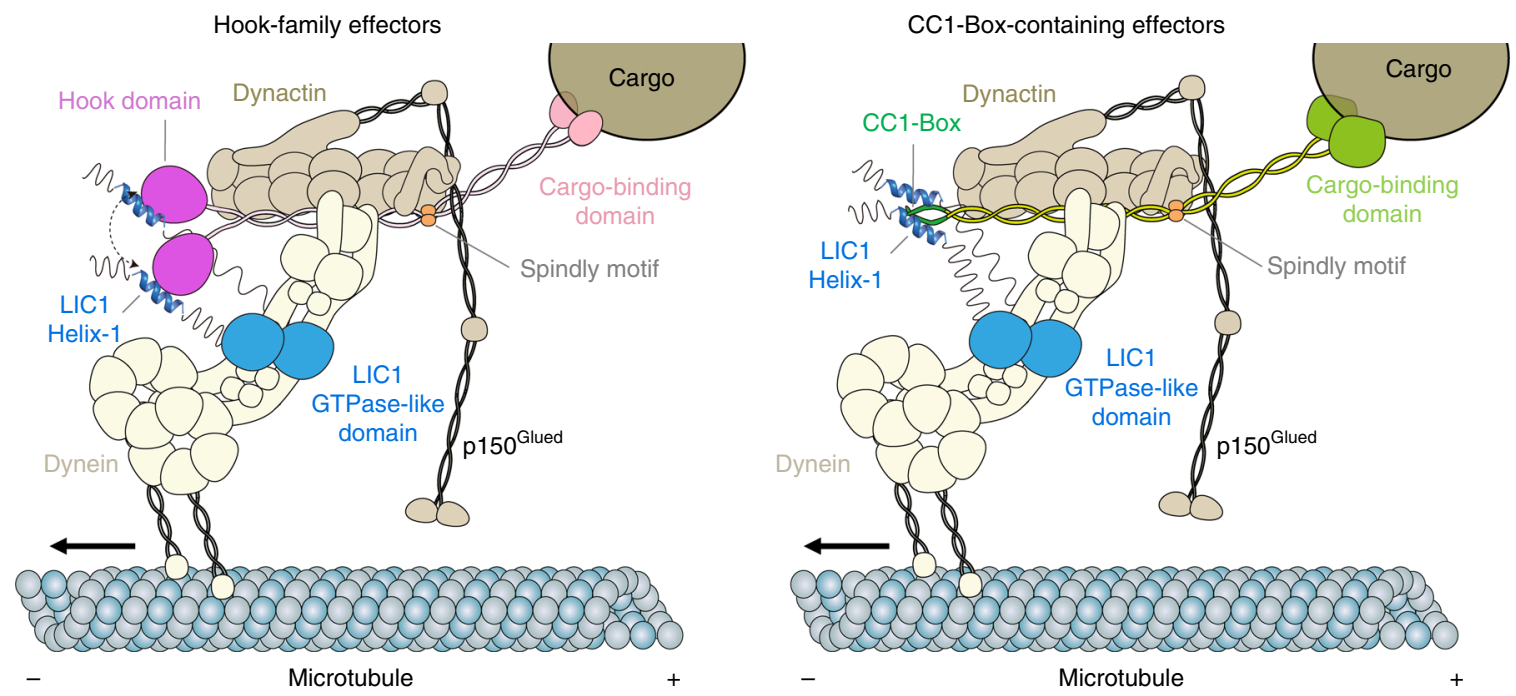

Fig. 6 Model for cargo transport by dynein-dynactin-effector complexes. Dynein is a 1.4 MDa homodimeric complex of two heavy chains, which each binds smaller subunits, including the intermediate chain, light intermediate chain, and three light chains. Dynactin is a 1.0 MDa complex of more than 20 proteins, including an actin filament-like core of actin-related protein 1 (Arp1) subunits, capped at both ends by several subunits, and a 'shoulder' domain from which emerges the largest subunit, p150 Glued, which projects $\sim 50 \mathrm{~nm}$ and can bind microtubules directly to help initiate a processive run. Although dynein and dynactin bind directly to each other via the dynein intermediate chain and the dynactin p150 Glued subunit ${ }^{30-33}$, they form a stable processive complex only in the presence of effector proteins, including Hook1/3 $3^{5,8,9}$, BICD2 $^{5-7}$, Spindly ${ }^{5}$, FIP3 $3^{5}$, and NIN/NINL ${ }^{10}$. These effectors are unrelated to each other in sequence and recruit different cargoes. Here we have demonstrated that independent of these differences, they all appear to interact with the same region of the dynein LIC1 subunit, which we named Helix-1 (or Effector-Binding Helix, EBH). The interaction involves the Hook domain in Hook-family effectors (left) or a coiled-coil segment in CC1-Box-containing effectors, such as BICD and Spindly (right). We have proposed here that the LIC1-effector interaction may help stabilize the 'parallel-heads' conformation thought to be necessary for dynein processivity ${ }^{16}$

the Hook domain (absent in the $\mathrm{CH}$ fold) that mediates the interaction by forming a V-shaped hydrophobic cleft after splitting into two helices. In BICD2 and Spindly, the interaction involves the CC1-Box, which forms part of a longer coiled-coil segment (Fig. 4a). Conceivably, the two helices of the coiled-coil could separate, partially exposing the hydrophobic core of the coiled-coil to create symmetric binding sites for LIC1 Helix-1 on both sides of the coiled-coil. This would give rise to a binding site that is different in sequence, but possibly structurally similar to that of the Hook domain.

The effectors analyzed here bind LIC1 with 1:1 stoichiometry, or rather with 2:2 stoichiometry, since all the effectors identified to date form dimers. In this way, each effector could in principle tether two LIC1 subunits from a single dynein dimer or from two different dynein dimers bound simultaneously to the dynactin complex. In the case of Hook-family effectors, the two LIC1binding sites are physically separated from one another, as suggested by our rotary shadowing EM analysis (Fig. 1h, i), whereas in CC1-Box-containing effectors the two binding sites occur on the same coiled-coil, i.e., adjacent to each other. Such structural differences, as well as differences in the affinities of the LIC1effector interactions, may play a modulatory role, by forming dynein-dynactin-effector complexes of different affinities and characterized by different run lengths. In this regard, it is important to note that the dynein-dynactin-Hook3 complex displays a bimodal velocity distribution and faster velocities ${ }^{8}$ than the dynactin-BICD2 complex characterized by a single velocity distribution ${ }^{5,6}$

The activation of dynein processivity proceeds through a conformational change from an auto-inhibited so-called 'phiparticle' state to a 'parallel-heads' state capable of binding microtubules upon complex formation with dynactin effectors ${ }^{16}$. The auto-inhibited state is stabilized by inter-heavy chain interactions, including near the LIC1 subunit. Because, the LIC1effector interaction appears to 'pull' on the LIC1 subunit and with it on the dynein heavy chain, we propose that it may help reposition the dynein heads for optimal interaction with microtubules by breaking the interaction between heavy chains (Fig. 6), and, thus, this interaction would be specifically important for the activation of dynein processivity. It remains to be demonstrated whether the LIC1-effector interaction is engaged at all times and whether it is absolutely required for dynein-dynactin complex formation, which could primarily depend on the other two interactions mentioned above (i.e., direct dynein-dynactin contacts and contacts mediated by an $\mathrm{N}$-terminal coiled-coil segment of the effectors).

\section{Methods}

Proteins. The cDNA encoding for human Hook1 (UniProt: Q9UJC3-1) and Hook3 (UniProt: Q86VS8-1) were purchased from Open Biosystems (Huntsville, AL). Constructs Hook $1_{11-166}$, Hook $1_{11-238}$, and Hook $1_{11-443}$ were cloned between BamHI and SalI sites of vector pMAL-c2x (NEB, Ipswich, MA). All the primers used in cloning are listed in Supplementary Table 1 . Construct Hook $1_{1-239}$ GCN4 was obtained by adding a 28-a.a. GCN4 sequence (MKQLEDKVEELLSKNYHLENEVARLKKL) by overlapping primers, while respecting the coiled-coil heptad register. The fusion construct was cloned as above. Constructs Hook $3_{1-143}$ and Hook $3_{1-160}$ were cloned between NotI and SalI sites of a modified pMAL-c2x (NEB) vector in which the Sacl site after MBP residue N367 was replaced with a NotI site. Point mutations A138D and M140D in Hook3 $3_{1-160}$ were introduced using the QuikChange site-directed mutagenesis kit (Agilent Technologies, Wilmington, DE). All the proteins were expressed in E. coli BL21 (DE3) cells (Invitrogen, Carlsbad, CA), grown in Terrific Broth medium at $37^{\circ} \mathrm{C}$ until the $\mathrm{OD}_{600}$ reached a value of $1.5-2$, followed by $16 \mathrm{~h}$ at $19^{\circ} \mathrm{C}$ in the presence of $0.25 \mathrm{mM}$ isopropyl- $\beta$-D-thiogalactoside. The cells were collected by centrifugation, resuspended in $20 \mathrm{mM}$ Tris, $\mathrm{pH} 7.0,100 \mathrm{mM} \mathrm{NaCl}, 4 \mathrm{mM}$ benzamidine hydrochloride, $1 \mathrm{mM}$ PMSF, and $1 \mathrm{mM}$ DTT and lysed using a Microfluidizer large-scale homogenizer (Microfluidics, Newton, MA). All the proteins were purified through an amylose affinity column according to the manufacturer's protocol (NEB). The MBP tag was removed by incubation with TEV protease overnight at $4{ }^{\circ} \mathrm{C}$. All the proteins were additionally purified by gel filtration on a SD200HL 26/60 column (GE Healthcare, Little Chalfont, UK) in $20 \mathrm{mM}$ Tris, pH 7.0, $100 \mathrm{mM} \mathrm{NaCl}, 1 \mathrm{mM}$ DTT.

The cDNA encoding for human LIC1 (UniProt: Q9Y6G9-1) was a generous gift from Ronald Vale (UCSF). Constructs $\mathrm{LICl}_{\mathrm{FL}}, \mathrm{LICl}_{1-461}$, and $\mathrm{LICl}_{1-437}$ were 
amplified by PCR and cloned between the BamHI and SalI sites of a modifiedvector pMAL-c2x that adds a C-terminal Strep-tag to the target protein. Point mutations F447A and F448A in $\mathrm{LIC1}_{\mathrm{FL}}$ were introduced using the QuikChange site-directed mutagenesis kit. Proteins were expressed and purified as described above, with one exception; after amylose affinity purification, the proteins were loaded onto a StrepTactin Sepharose column (IBA Lifesciences, Göttingen, Germany) and eluted after extensive washing with $3 \mathrm{mM}$ desthiobiotin, $20 \mathrm{mM}$ Tris, $\mathrm{pH} 7.0,100 \mathrm{mM} \mathrm{NaCl}$, and $1 \mathrm{mM}$ DTT. To obtain the Helix-1 peptides, the cDNA encoding for $\mathrm{LICl}_{433-458}$ (Fig. 2a) was cloned between the SapI and SalI sites of vector pTYB11 (NEB). Point mutations F447A and F448A were introduced using the QuikChange site-directed mutagenesis kit to obtain the mutant peptide Helix- $1_{\mathrm{F} 447 \mathrm{~A}, \mathrm{~F} 448 \mathrm{~A}}$. Proteins were expressed as above, and purified on a chitin affinity column according to the manufacturer's protocol (NEB), followed by autocleavage of the intein tag induced by incubation with $50 \mathrm{mM}$ DTT overnight at $4^{\circ}$ C. The cleaved peptides were additionally purified on a Symmetry300 $\mathrm{C}_{18}$ reversephase column (Waters, Milford, MA) using an acetonitrile gradient of $0-90 \%(\mathrm{v} / \mathrm{v})$ and $0.1 \%(\mathrm{v} / \mathrm{v})$ trifluoroacetic acid.

The cDNA encoding for full-length human Hook1 was codon optimized for expression in Sf9 cells and synthesized (Genscript Biotech, Piscataway, NJ). The gene was cloned between SalI and XbaI sites of a modified-vector pFastBacl, which adds a V5 epitope tag at the $\mathrm{N}$ terminus and a Strep-tag at the C-terminal of the target protein. The protein was expressed in Spodoptera frugiperda 9 (Sf9) cells using the Bac-to-Bac baculovirus expression system according to the manufacturer's protocol (Invitrogen). The cells were collected by centrifugation, resuspended in lysis buffer $\left(10 \mathrm{mM} \mathrm{Na} \mathrm{HPO}_{4}, \mathrm{pH} 7.4,100 \mathrm{mM} \mathrm{NaCl}, 1 \mathrm{mM} \mathrm{PMSF}\right.$, $4 \mathrm{mM}$ Benzamidine, $1 \mathrm{mM}$ DTT, and $5 \%$ glycerol $(\mathrm{v} / \mathrm{v}))$ with addition of a protease inhibitor cocktail (Roche, Basel, Switzerland). The cells were lysed by addition of $0.5 \%(\mathrm{v} / \mathrm{v})$ Triton X-100 through three cycles of freeze-thaw on ice and centrifuged $20 \mathrm{~min}$ at $20,000 \times g$. Lysates were loaded onto a StrepTactin Sepharose column, and after washing extensively with lysis buffer, Hook $1_{\mathrm{FL}}$ was eluted with the addition of $3 \mathrm{mM}$ desthiobiotin. The protein was additionally purified through a Superose 6 gel filtration column (GE Healthcare), equilibrated with $20 \mathrm{mM}$ Tris, $\mathrm{pH} 7.5,100 \mathrm{mM} \mathrm{NaCl}$, and $1 \mathrm{mM}$ DTT.

The cDNAs encoding for human BICD2 ${ }_{1-98}$ (UniProt: Q8TD16-1) and Spindly ${ }_{1-142}$ (UniProt: Q96EA4-1) were synthesized with codon optimization for $E$. coli expression (Genscript Biotech). The BICD2 $2_{1-98}$ gene was cloned between the BamHI and SalI sites of vector pMAL-c2x. The protein was expressed and purified as described above. The Spindly ${ }_{1-142}$ gene was cloned between the BamHI and SalI sites of vector pCold1 (TAKARA BIO, Kusatsu, Japan). The protein was expressed in BL21 (DE3) cells as described above. The protein was purified through a NiNTA affinity column in $50 \mathrm{mM}$ Tris, $\mathrm{pH} 8.0,500 \mathrm{mM} \mathrm{NaCl}, 4 \mathrm{mM}$ benzamidine hydrochloride, and $1 \mathrm{mM}$ PMSF and eluted with $250 \mathrm{mM}$ Imidazole. The $\mathrm{His}_{6}$-tag was removed by overnight incubation with TEV protease at $4{ }^{\circ} \mathrm{C}$, and the protein was additionally purified through a HiLoad $16 / 600$ Superdex 75 pg column (GE Healthcare) in $20 \mathrm{mM}$ Tris $\mathrm{pH} 7.0,100 \mathrm{mM} \mathrm{NaCl}$, and $1 \mathrm{mM}$ DTT.

Multi-angle light scattering. Samples were separated by size exclusion chromatography on a Superose 6 10/300 GL column (GE Healthcare) equilibrated with 20 $\mathrm{mM}$ Tris, $\mathrm{pH} 7.5,100 \mathrm{mM} \mathrm{NaCl}$, and $1 \mathrm{mM}$ DTT, using an Agilent 1100 HPLC system (Agilent Technologies). Multi-angle light scattering was measured in line using a DAWN-HELEOS Multi-angle light scattering detector and an Optilab rEX refractive index detector. The scattering data were analyzed with the ASTRA software (Wyatt Technology, Santa Barbara, California).

Isothermal titration calorimetry. ITC measurements were carried out on a VPITC instrument (MicroCal, Northampton, MA). Protein samples were dialyzed for $2 \mathrm{~d}$ against $20 \mathrm{mM}$ HEPES, pH 7.5, $100 \mathrm{mM} \mathrm{NaCl}$, and $0.25 \mathrm{mM}$ TCEP (ITC buffer). The $\mathrm{LIC1}_{433-458}$ peptide was re-suspended in ITC buffer, followed by three cycles of lyophilization/resolubilization in $50 \%(\mathrm{v} / \mathrm{v})$ methanol to remove any trifluoroacetic acid remaining after reverse-phase purification. The peptide was then re-suspended in ITC buffer. The proteins (or $\mathrm{LICl}_{433-458}$ peptide) in the syringe were titrated at a concentration 10 - to 20 -fold higher than that of the proteins in the ITC cell of total volume $1.44 \mathrm{ml}$ (as indicated in the figures). Titrations consisted of $10 \mu \mathrm{l}$ injections, lasting for $10 \mathrm{~s}$, with an interval of 300-400 s between injections. The heat of binding was corrected for the heat of injection, determined by injecting proteins into buffer. Data were analyzed using the program Origin (OriginLab, Northampton, MA). The temperature and parameters of the fit (stoichiometry and affinity) of each experiment are given in the figures.

Rotary shadowing and electron microscopy. Full-length Hook1 was suspended in a solution containing $10 \mathrm{mM}$ Tris, $\mathrm{pH} 7.5,50 \mathrm{mM} \mathrm{NaCl}, 1 \mathrm{mM}$ DTT, and $50 \%$ $(\mathrm{v} / \mathrm{v})$ glycerol. Samples were diluted to a concentration of $100 \mu \mathrm{g} / \mathrm{ml}$ in the same solution and $1 \mu \mathrm{g}$ of each sample were sprayed on a freshly split mica surface and dried for $1 \mathrm{~h}$ at room temperature. The samples were rotary shadowed with platinum at a $7^{\circ}$ angle, and replicated with carbon in a Balzers 410 freeze-fracture machine. Replicas were photographed at a magnification of 98,900 using a Philips 410 transmission electron microscope operating at $80 \mathrm{kV}$. The original images were obtained from areas at the edge of each droplet that showed distinct nonaggregated molecules and a clear background. Images were analyzed using the Image software ${ }^{35}$
Crystallization and structure determination. Hook $3_{1-160}$ at $10 \mathrm{mg} / \mathrm{ml}$ in $10 \mathrm{mM}$ Tris, $\mathrm{pH} 7.4,25 \mathrm{mM} \mathrm{NaCl}$, and $2 \mathrm{mM}$ TCEP was mixed with 1.2 molar excess of the $\mathrm{LICl}_{433-458}$ peptide at $4{ }^{\circ} \mathrm{C}$ for $1 \mathrm{~h}$. Crystal were obtained at $20^{\circ} \mathrm{C}$ using the hanging-drop method. The crystallization drop consisted of a 1:1 (v/v) mixture of protein solution and well solution (1.44 M ammonium citrate tribasic, $\mathrm{pH}$ 6.25). The crystals were improved through consecutive rounds of micro-seeding. For data collection, the crystals were flash-frozen in liquid nitrogen from a cryo-solution consisting of crystallization buffer with addition of $30 \%(\mathrm{v} / \mathrm{v})$ glycerol.

An x-ray diffraction dataset was collected at the Cornell High Energy Synchrotron Source (CHESS) beamline F1. The diffraction data were indexed and scaled using the program HKL2000 $0^{36}$. A molecular replacement solution was obtained with the program Phenix ${ }^{37}$ using PDB entry 5J8E (unbound Hook domain of Hook $3^{9}$ ). Model building and refinement were carried out with the programs $\operatorname{Coot}^{38}$ and Phenix ${ }^{37}$. Figures were generated with the program PyMOL (Schrödinger, New York City, NY). Sequence alignments were carried out with the program MAFFT ${ }^{39}$ and visualized using ESPript ${ }^{40}$. Data collection and refinement statistics are listed in Table 1.

Single-molecule motility assays. The motility of dynein-dynactin-Hook $3_{1-552}$ or dynein-dynactin-BICD2 $2_{1-572}$ complexes from cell extracts were tracked using TIRF microscopy ${ }^{8}$. The motility assays were performed in flow chambers constructed with a glass slide and a silanized (PlusOne Repel Silane, GE Healthcare) coverslip, held together with double sided adhesive tape and vacuum grease to form a $\sim 15 \mu \mathrm{l}$ chamber. A 1:40 dilution of monoclonal anti- $\beta$-tubulin antibody (T5201, Sigma) was perfused into the chamber, which was subsequently blocked with $5 \%$ pluronic F-127 (Sigma-Aldrich, St. Louis, MO). Taxol-stabilized microtubules, labeled with HiLyte 488 or 647 (Cytoskeleton, Denver, CO) at a labeling ratio of 1:40, were flowed into the chamber and immobilized by interaction with anti- $\beta$-tubulin antibodies.

HeLa cells expressing Halo-tagged Hook $3_{1-552}$ or BICD2 $2_{1-572}$ were labeled with TMR-HaloTag ligand (Promega, Madison, WI) $18-20 \mathrm{~h}$ post-transfection. Cells grown in $10 \mathrm{~cm}$ plates to $70-80 \%$ confluence were then lysed in $100 \mu \mathrm{l}$ lysis buffer (40 mM HEPES pH 7.4, $120 \mathrm{mM} \mathrm{NaCl}, 1 \mathrm{mM}$ EDTA, $1 \mathrm{mM} \mathrm{Mg-ATP,} 0.1 \%$ Triton X-100, $1 \mathrm{mM}$ PMSF, $0.01 \mathrm{mg} / \mathrm{ml}$ TAME, $0.01 \mathrm{mg} / \mathrm{ml}$ leupeptin and $1 \mu \mathrm{g} / \mathrm{ml}$ pepistatin-A). Cell lysates were then clarified by centrifugation at 17,000x $g$. Before flowing into the imaging chamber, the cell extracts were diluted in P12 buffer (12 mM PIPES, pH 6.8, $1 \mathrm{mM}$ EGTA, $2 \mathrm{mM} \mathrm{MgCl}_{2}$, and $20 \mu \mathrm{M}$ Taxol). The cells lysates were then further diluted in motility buffer $(1 \times \mathrm{P} 12$ buffer supplemented with $10 \mathrm{mM} \mathrm{Mg}$-ATP, $0.3 \mathrm{mg} / \mathrm{ml}$ casein, $0.3 \mathrm{mg} / \mathrm{ml}$ bovine serum albumin, and 10 $\mathrm{mM} \mathrm{DTT})$ and an oxygen scavenging system $(0.5 \mathrm{mg} / \mathrm{ml}$ glucose oxidase, $470 \mathrm{U} / \mathrm{ml}$ catalase and $15 \mathrm{mg} / \mathrm{ml}$ glucose) and flowed into the chamber to be imaged.

The dynein-driven motility of single Halo-Hook $3_{1-552}$ or Halo-BICD2 $2_{1-572}$ positive molecules was then examined in the absence or the presence of varying concentrations of the Helix-1 (or Helix- $1_{\mathrm{F} 447 \mathrm{~A}, \mathrm{~F} 448 \mathrm{~A}}$ ) peptide, added to the motility buffer immediately prior to the addition of the cell lysates. All the movies (4 frames/s) were acquired at room temperature using a Nikon TIRF system (Perkin Elmer, Waltham, MA) on an inverted Ti microscope equipped with a $100 \times$ objective and an ImageEM C9100-13 camera (Hamamatsu Photonics, Hamamatsu City, Japan) controlled by Volocity software (Improvision, Lexington, MA). Particle tracking was performed using the TrackMate plugin in the program Fiji ${ }^{41}$ Particle runs were tracked only if both the start and end of a run were observable over the course of the movie. Runs on microtubule bundles were excluded from this analysis.

Lysosomal distribution analysis. HeLa cells transfected for $18-22 \mathrm{~h}$ with GFP, $\mathrm{LIC1}_{\mathrm{WT}}$-GFP, or $\mathrm{LIC1}_{\mathrm{F} 447 \mathrm{~A}, \mathrm{~F} 448 \mathrm{~A}}$-GFP were fixed with PFA. Cells were then stained with anti-LAMP1 antibodies (rabbit polyclonal, ab24170, Abcam, Cambridge, UK) diluted 1:500, followed by anti-rabbit secondary antibodies, and mounted on glass coverslips with ProLong Gold anti-fade reagent (Invitrogen). Images were taken with an inverted epifluorescence microscope (DMI6000B, Leica Camera AG, Wetzlar, Germany) using an Apochromat $63 \times, 1.4$ NA oil immersion objective (Leica Camera AG).

The images were blinded before analysis, and the distribution of LAMP1positive vesicles was scored using cells expressing GFP only. LAMP1 staining was scored "abnormal" if the cells lacked the characteristic perinuclear lysosomal clustering and/or had several or a few enlarged LAMP1-positive vesicles dispersed throughout the cell.

Data availability. Atomic coordinates and structure factor amplitudes for the crystal structure of the Hook3-LIC1 complex were deposited with the Protein Data Bank (PDB) under accession code $6 \mathrm{~B} 9 \mathrm{H}$. Other data and materials are available from the corresponding author upon reasonable request.

Received: 14 November 2017 Accepted: 12 February 2018 Published online: 07 March 2018 


\section{References}

1. Barlan, K. \& Gelfand, V. I. Microtubule-based transport and the distribution, tethering, and organization of organelles. Cold Spring Harb. Perspect. Biol. 9, a025817 (2017).

2. Bonifacino, J. S. \& Neefjes, J. Moving and positioning the endolysosomal system. Curr. Opin. Cell Biol. 47, 1-8 (2017).

3. Hoogenraad, C. C. SpringerAmpamp; Akhmanova, A. Bicaudal D family of motor adaptors: linking dynein motility to cargo binding. Trends Cell Biol. 26, 327-340 (2016).

4. Carter, A. P., Diamant, A. G. \& Urnavicius, L. How dynein and dynactin transport cargos: a structural perspective. Curr. Opin. Struct. Biol. 37, 62-70 (2016).

5. McKenney, R. J., Huynh, W., Tanenbaum, M. E., Bhabha, G. \& Vale, R. D. Activation of cytoplasmic dynein motility by dynactin-cargo adapter complexes. Science 345, 337-341 (2014).

6. Schlager, M. A., Hoang, H. T., Urnavicius, L., Bullock, S. L. \& Carter, A. P. In vitro reconstitution of a highly processive recombinant human dynein complex. Embo. J. 33, 1855-1868 (2014).

7. Splinter, D. et al. BICD2, dynactin, and LIS1 cooperate in regulating dynein recruitment to cellular structures. Mol. Biol. Cell. 23, 4226-4241 (2012).

8. Olenick, M. A., Tokito, M., Boczkowska, M., Dominguez, R. \& Holzbaur, E. L. Hook adaptors induce unidirectional processive motility by enhancing the dynein-dynactin interaction. J. Biol. Chem. 291, 18239-18251 (2016).

9. Schroeder, C. M. \& Vale, R. D. Assembly and activation of dynein-dynactin by the cargo adaptor protein Hook3. J. Cell Biol. 214, 309-318 (2016).

10. Redwine, W. B. et al. The human cytoplasmic dynein interactome reveals novel activators of motility. eLife 6, e28257 (2017).

11. Schroeder, C. M., Ostrem, J. M., Hertz, N. T. \& Vale, R. D. A Ras-like domain in the light intermediate chain bridges the dynein motor to a cargo-binding region. eLife 3, e03351 (2014).

12. Gama, J. B. et al. Molecular mechanism of dynein recruitment to kinetochores by the Rod-Zw10-Zwilch complex and Spindly. J. Cell Biol. 216, 943-960 (2017).

13. Maldonado-Báez, L., Cole, N. B., Krämer, H. \& Donaldson, J. G. Microtubuledependent endosomal sorting of clathrin-independent cargo by Hook1. J. Cell Biol. 201, 233-247 (2013).

14. Xu, L. et al. An FTS/Hook/p107 ${ }^{\text {FHIP }}$ complex interacts with and promotes endosomal clustering by the homotypic vacuolar protein sorting complex. Mol. Biol. Cell 19, 5059-5071 (2008).

15. Luiro, K. et al. Interconnections of CLN3, Hook1 and Rab proteins link Batten disease to defects in the endocytic pathway. Hum. Mol. Genet. 13, 3017-3027 (2004).

16. Zhang, K. et al. Cryo-EM reveals how human cytoplasmic dynein is autoinhibited and activated. Cell 169, 1303-1314 (2017).

17. Jordens, I. et al. The Rab7 effector protein RILP controls lysosomal transport by inducing the recruitment of dynein-dynactin motors. Curr. Biol. 11 1680-1685 (2001).

18. Johansson, M. et al. Activation of endosomal dynein motors by stepwise assembly of Rab7-RILP-p150Glued, ORP1L, and the receptor blll spectrin. J. Cell Biol. 176, 459-471 (2007).

19. Tan, S. C., Scherer, J. \& Vallee, R. B. Recruitment of dynein to late endosomes and lysosomes through light intermediate chains. Mol. Biol. Cell 22, 467-477 (2011).

20. Trokter, M., Mücke, N. \& Surrey, T. Reconstitution of the human cytoplasmic dynein complex. Proc. Natl Acad. Sci. USA 109, 20895-20900 (2012).

21. Kardon, J. R. \& Vale, R. D. Regulators of the cytoplasmic dynein motor. Nat. Rev. Mol. Cell Biol. 10, 854-865 (2009).

22. Hoogenraad, C. C. et al. Mammalian golgi-associated Bicaudal-D2 functions in the dynein-dynactin pathway by interacting with these complexes. EMBO J. 20, 4041-4054 (2001).

23. Bielska, E. et al. Hook is an adapter that coordinates kinesin-3 and dynein cargo attachment on early endosomes. J. Cell Biol. 204, 989-1007 (2014).

24. Sano, H. et al. The microtubule-binding protein Hook3 interacts with a cytoplasmic domain of scavenger receptor A. J. Biol. Chem. 282, 7973-7981 (2007).

25. Horgan, C. P., Hanscom, S. R., Jolly, R. S., Futter, C. E. \& McCaffrey, M. W. Rab11-FIP3 links the Rab11 GTPase and cytoplasmic dynein to mediate transport to the endosomal-recycling compartment. J. Cell Sci. 123, 181-191 (2010).

26. Chowdhury, S., Ketcham, S. A., Schroer, T. A. \& Lander, G. C. Structural organization of the dynein-dynactin complex bound to microtubules. Nat. Struct. Mol. Biol. 22, 345-347 (2015).

27. Urnavicius, L. et al. The structure of the dynactin complex and its interaction with dynein. Science 347, 1441-1446 (2015).

28. Urnavicius, L. et al. Cryo-EM shows how dynactin recruits two dyneins for faster movement. Nature 554, 202-206 (2018).

29. Grotjahn, D. A. et al. Cryo-electron tomography reveals that dynactin recruits a team of dyneins for processive motility. Nat. Struct. Mol. Biol. doi: 10.1038/ s41594-018-0027-7 (2018).
30. Karki, S. \& Holzbaur, E. L. Affinity chromatography demonstrates a direct binding between cytoplasmic dynein and the dynactin complex. J. Biol. Chem. 270, 28806-28811 (1995)

31. Vaughan, K. T. \& Vallee, R. B. Cytoplasmic dynein binds dynactin through a direct interaction between the intermediate chains and p150 Glued. J. Cell Biol. 131, 1507-1516 (1995).

32. Morgan, J. L., Song, Y. \& Barbar, E. Structural dynamics and multiregion interactions in dynein-dynactin recognition. J. Biol. Chem. 286, 39349-39359 (2011).

33. Siglin, A. E. et al. Dynein and dynactin leverage their bivalent character to form a high-affinity interaction. PLOS ONE 8, e59453 (2013).

34. Rao, L. et al. The yeast dynein Dyn2-Pac11 complex is a dynein dimerization/ processivity factor: structural and single-molecule characterization. Mol. Biol. Cell 24, 2362-2377 (2013)

35. Schneider, C. A., Rasband, W. S. \& Eliceiri, K. W. NIH Image to ImageJ: 25 years of image analysis. Nat. Methods 9, 671-675 (2012).

36. Otwinowski, Z. \& Minor, W. Processing of X-ray diffraction data collected in oscillation mode. Methods Enzymol. 276, 307-326 (1997).

37. Adams, P. D. et al. PHENIX: a comprehensive Python-based system for macromolecular structure solution. Acta Crystallogr. D 66, 213-221 (2010).

38. Emsley, P., Lohkamp, B., Scott, W. G. \& Cowtan, K. Features and development of Coot. Acta Cryst. 66, 486-501 (2010).

39. Katoh, K., Misawa, K., Kuma, Ki \& Miyata, T. MAFFT: a novel method for rapid multiple sequence alignment based on fast Fourier transform. Nucleic Acids Res. 30, 3059-3066 (2002).

40. Robert, X. \& Gouet, P. Deciphering key features in protein structures with the new ENDscript server. Nucleic Acids Res. 42, W320-W324 (2014).

41. Schindelin, J. et al. Fiji: an open-source platform for biological-image analysis Nat. Methods 9, 676-682 (2012).

42. Chan, Y. W. et al. Mitotic control of kinetochore-associated dynein and spindle orientation by human Spindly. J. Cell Biol. 185, 859-874 (2009).

43. Wong, Y. C. \& Holzbaur, E. L. The regulation of autophagosome dynamics by huntingtin and HAP1 is disrupted by expression of mutant huntingtin, leading to defective cargo degradation. J. Neurosci. 34, 1293-1305 (2014).

44. van Spronsen, M. et al. TRAK/Milton motor-adaptor proteins steer mitochondrial trafficking to axons and dendrites. Neuron 77, 485-502 (2013).

\section{Acknowledgements}

This work was supported by National Institutes of Health Grants P01 GM087253 to R.D. (Project 3) and E.L.F.H. (Project 4) and R01 GM073791 to R.D.

\section{Author contributions}

I.-G.L., E.L.F.H., and R.D. designed the experiments. I.-G.L. performed the biochemical and structural experiments with M.B. and R.D.'s participation. M.A.O. performed the in vitro motility and cellular experiments. C.F.-A. performed the rotary shadowing EM experiments. I.-G.L. and R.D. wrote the manuscript. All the authors reviewed the figures and manuscript and approved its final version.

\section{Additional information}

Supplementary Information accompanies this paper at https://doi.org/10.1038/s41467018-03412-8.

Competing interests: The authors declare no competing interests.

Reprints and permission information is available online at http://npg.nature.com/ reprintsandpermissions/

Publisher's note: Springer Nature remains neutral with regard to jurisdictional claims in published maps and institutional affiliations.

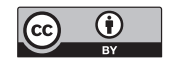

Open Access This article is licensed under a Creative Commons Attribution 4.0 International License, which permits use, sharing, adaptation, distribution and reproduction in any medium or format, as long as you give appropriate credit to the original author(s) and the source, provide a link to the Creative Commons license, and indicate if changes were made. The images or other third party material in this article are included in the article's Creative Commons license, unless indicated otherwise in a credit line to the material. If material is not included in the article's Creative Commons license and your intended use is not permitted by statutory regulation or exceeds the permitted use, you will need to obtain permission directly from the copyright holder. To view a copy of this license, visit http://creativecommons.org/ licenses/by/4.0/.

(C) The Author(s) 2018 